\title{
Ares Launch Vehicle Transonic Buffet Testing and Analysis Techniques
}

\author{
David J. Piatak ${ }^{1}$ Martin K. Sekula ${ }^{2}$ Russ D. Rausch ${ }^{3}$ \\ Aeroelasticity Branch / NASA Langley Research Center \\ Hampton, VA 23681
}

\begin{abstract}
It is necessary to define the launch vehicle buffet loads to ensure that structural components and vehicle subsystems possess adequate strength, stress, and fatigue margins when the vehicle structural dynamic response to buffet forcing functions are considered. In order to obtain these forcing functions, the accepted method is to perform wind-tunnel testing of a rigid model instrumented with hundreds of unsteady pressure transducers designed to measure the buffet environment across the desired frequency range. The buffet wind-tunnel test program for the Ares Crew Launch Vehicle employed 3.5 percent scale rigid models of the Ares I and Ares I-X launch vehicles instrumented with 256 unsteady pressure transducers each. These models were tested at transonic conditions at the Transonic Dynamics Tunnel at NASA Langley Research Center. The ultimate deliverable of the Ares buffet test program are buffet forcing functions (BFFs) derived from integrating the measured fluctuating pressures on the rigid wind-tunnel models. These BFFs are then used as input to a multi-mode structural analysis to determine the vehicle response to buffet and the resulting buffet loads and accelerations. This paper discusses the development of the Ares $I$ and I-X rigid buffet model test programs from the standpoint of model design, instrumentation system design, test implementation, data analysis techniques to yield final products, and presents normalized sectional buffet forcing function root-mean-squared levels.
\end{abstract}

\section{Nomenclature}

$\begin{array}{ll}A & =\text { Area } \\ \mathrm{BFF} & =\text { buffet forcing function } \\ \mathrm{BPF} & =\text { blade passage frequency } \\ D & =\text { diameter } \\ C M & =\text { crew module } \\ C_{p} & =\text { pressure coefficient } \\ C_{y} & =\text { force coefficient in the y direction } \\ C_{z} & =\text { force coefficient in the } z \text { direction } \\ f & =\text { frequency, Hz } \\ F & =\text { force } \\ F R F & =\text { frequency response function } \\ f_{x} & =X \text { component of sectional buffet forcing function } \\ f_{y} & =Y \text { component of sectional buffet forcing function } \\ f_{z} & =Z \text { component of sectional buffet forcing function } \\ F_{x} & =X \text { component of point load buffet forcing function } \\ F_{y} & =Y \text { component of point load buffet forcing function } \\ F_{z} & =Z \text { component of point load buffet forcing function } \\ L & =\text { length } \\ L A S & =\text { launch abort system } \\ p & =\text { pressure } \\ q & =\text { dynamic pressure, psf }\end{array}$

\footnotetext{
${ }^{1}$ Senior Aerospace Engineer, Aeroelasticity Branch, Mail Stop 340.

${ }^{2}$ Senior Aerospace Engineer, Aeroelasticity Branch, Mail Stop 340.

${ }^{3}$ Senior Aerospace Engineer, Aeroelasticity Branch, Mail Stop 340.
} 


$\begin{array}{ll}\text { RBM } & =\text { rigid buffet model } \\ R e & =\text { Reynolds number, per-foot or non-dimensional based on } 1^{\text {st }} \text { stage diameter } \\ r m s & =\text { root-mean-squared } \\ R P M & =\text { revolutions per minute } \\ S M & =\text { service module } \\ T & =\text { time } \\ \text { TDT } & =\text { Transonic Dynamics Tunnel } \\ V & =\text { velocity } \\ \Delta & =\text { indicates fluctuating component } \\ \phi & =\text { vehicle roll angle, deg } \\ \theta & =\text { vehicle pitch angle, deg }\end{array}$

\section{Notice to Readers}

The predicted performance and certain other features and characteristics of the Ares I and Ares I-X launch vehicles are defined by the U.S. Government to be Sensitive But Unclassified (SBU). Therefore, details have been removed from all plots and figures.

\section{Introduction}

$\mathrm{B}$ uffet is an unsteady aerodynamic phenomenon characterized by fluctuating pressures resulting from flowinduced turbulence, flow separation, wake effects, and shock oscillations. These fluctuating pressures can produce significant loads on a launch vehicle and spacecraft during ascent to orbit. When buffet occurs on a launch vehicle, the fluctuating pressure loads can excite vehicle bending modes and local shell/panel modes, as illustrated in Figure 1. The buffet environment is typically most extreme in the transonic regime as the vehicle approaches the speed of sound. At this point in the trajectory, shocks form on the vehicle and interact with other flow phenomena at locations where changes in the vehicle geometry occur. For buffet loads analysis of launch vehicles, the buffeting response is limited to the low frequency bending modes of the vehicle, which have frequencies that are typically below $60 \mathrm{~Hz}$. Higher frequency vibratory responses due to aeroacoustic excitation fall under the regime of vibroacoustic loads (refs. 1-3).

At this time, it is impractical to use computational fluid dynamics (CFD) to predict turbulent unsteady aerodynamic flow for buffet loads analysis of launch vehicles. Time accurate CFD solutions are deemed too costly to obtain for the wide range of conditions of interest. Consequently, experimental buffet pressure data is a necessity in attempting to understand and predict critical buffet loads. The accepted method of assessing vehicle buffeting response is to acquire experimental fluctuating pressure data of the buffet environment using a rigid model instrumented with hundreds of unsteady pressure transducers. The unsteady pressure data are developed into buffet forcing functions at stations along the vehicle and are used as input to a multi-mode structural analysis in order to assess the vehicle response and the resulting buffet loads and accelerations (refs. 4-6).

This paper discusses the test development, test implementation, and data analysis methodologies associated with the Ares Crew Launch Vehicle (CLV) buffet test program. Two buffet wind-tunnel tests have been conducted on rigid buffet models (RBMs) of first the Ares I-X followed by the Ares I launch vehicle. These tests were conducted at NASA Langley's Transonic Dynamics Tunnel (TDT) for the purpose of ascertaining ascent buffet and aeroacoustic environments of the vehicles for detailed structural/aerodynamic design. Fluctuating buffet pressure data have been acquired at 256 locations on the surface of both models that provide adequate spatial resolution to resolve the buffet environment. A subset of sensors was allocated to acquire aeroacoustic bandwidth fluctuating pressures. Test conditions varied from Mach 0.5 to 1.2 with focus on the Mach 0.8 to 1.0 regime. Model pitch and roll angles ranged between \pm 8 degrees and \pm 180 degrees, respectively. To acquire this large volume of space-timedependent data, three separate data acquisition systems with overlapping sampling bandwidths were employed to ensure adequate spectral resolution for both the buffet and aeroacoustic pressure fluctuations (ref. 7-8). Analysis methods associated with the integration of pressures yielded buffet forcing functions, development of correlation lengths from longitudinal coherence analyses, and scaling of the model-scale data to full-scale trajectory flight conditions are described in reference 9 and in the present paper. Finally, this paper presents and compares the buffet forcing functions obtained from Ares I and I-X buffet testing to illustrate the results of these tests and the differences in the buffet environments on these vehicles (refs. 10-11). 


\section{Test Objectives}

The primary test objective was to acquire time-correlated unsteady pressure data up to a minimum frequency of $60 \mathrm{~Hz}$ full-scale on 3.5 percent scale Ares I and I-X RBMs at transonic conditions. The secondary test objective was to simultaneously acquire aeroacoustic unsteady pressure time histories up to a frequency of 2,000 $\mathrm{Hz}$ full-scale on a limited number of sensors. Test condition variables included tunnel conditions (Mach number and dynamic pressure), model pitch angle, and model roll angle. Model test configurations included both a clean model and the model with all vehicle protuberances. Deliverables to the Ares and Constellation Programs from this buffet test program include buffet forcing function databases for use in vehicle coupled loads analysis. The buffet forcing function database contains orthogonal point load time histories resulting from measured buffet pressures along the length of the vehicle at key trajectory and vehicle conditions.

\section{Test Facility}

The NASA Langley Transonic Dynamics Tunnel has a 16-foot-by-16-foot slotted test section capable of reaching Mach 1.2. It is a closed-circuit, continuous flow wind tunnel built for the purposes of conducting aeroelastic testing. The TDT is capable of testing over a range of stagnation pressures from near vacuum to atmospheric and Mach numbers from near zero to 1.2 in both air and a heavy gas test medium (R134a). Dynamic pressures up to 300 psf and 550 psf can be attained in an air and R134a test medium, respectively. Table 1 lists test conditions for several Mach numbers for the Ares RBM test program. Past launch vehicle tests conducted at TDT include Apollo-Saturn, Atlas-Centaur, and Delta-series tests. (refs. 12-14)

Testing in a heavy gas is advantageous to test programs where time and frequency scaling are of importance, such as for launch vehicle buffet and aeroacoustic testing. Since the speed of sound in R134a is approximately half that in air, model-scale frequencies in R134a are also half those in air, resulting in more favorable bandwidth requirements for instrumentation and data systems.

\section{Model Design}

The need to house 256 unsteady pressure transducers and their associated cabling and tubing necessitated the model be significantly larger than force and moment models tested as part of the Ares program. A larger model is also advantageous from the standpoint of time and frequency scaling in that the model-scale frequency bandwidth is inversely proportional to the model geometric scale factor. Thus, a larger model yields a lower model-scale bandwidth requirement for transducers and data systems. Another consideration in choosing the geometric scale factor for a launch vehicle rigid buffet model is the choice of test facility. Two test facilities were targeted by the Ares buffet test program: the TDT and the Arnold Engineering Development Center's 16T Propulsion Wind Tunnel, both with 16-ft transonic test sections. Based on the lengths of these test sections, a 3.5 percent geometric scale was chosen for the buffet model test program. Figure 2 shows the Ares I-X RBM installed in the TDT test section and Figures 3 and 4 show the Ares I RBM.

The scaling of time and frequency are important in the design of a rigid buffet model to assure that the model, test medium, and test instrumentation are capable of resolving the expected unsteady pressure levels and the bandwidth desired. Table 2 contains scaling laws based on similitude parameters for Strouhal scaling used in the design of the Ares I and I-X RBMs.

\section{Instrumentation}

Pressure instrumentation for Ares RBM testing must be capable of resolving both the steady component of pressure plus the fluctuating flow field at a model-scale bandwidth of up to $900 \mathrm{~Hz}$ to capture the buffet bandwidth up to $60 \mathrm{~Hz}$ full-scale. A subset of this instrumentation has more stringent model-scale bandwidth requirements of up to $30 \mathrm{KHz}$ in an R134a test medium to acquire aeroacoustic data with a bandwidth up to 2,000 $\mathrm{Hz}$ full-scale. Table 1 lists the minimum instrumentation bandwidth requirements at several Mach numbers for buffet and aeroacoustic objectives for the Ares RBM test program.

Kulite "leadless" 093- and 072-style fluctuating pressure transducers with integrated amplifiers were chosen for use in the Ares RBM test program based on previous test experience with this transducer design and its ability to meet test instrumentation requirements. For both the Ares I and I-X RBMs, 256 pressure transducers were located at 46 and 52 longitudinal vehicle stations, respectively. Pressure transducers were placed in azimuthal rings of four or eight sensors at each longitudinal vehicle station. Distribution of pressure transducers was guided by steady computational fluid dynamics (CFD) results with emphasis on regions of high pressure gradients and other locations 
where fluctuating buffet pressures would be expected. Figure 5 presents pressure transducer locations for the Ares I RBM (also representative of the sensor distribution of the Ares I-X RBM).

In addition to unsteady pressure transducers, six accelerometers were located within the models to obtain model vibrations and three Q-flex accelerometers were positioned near the frustum for model pitch and roll orientation. Figure 6 shows internal details of the Ares I RBM including pressure transducer amplifiers, instrumentation cabling, and reference pressure tubing. Based on lessons learned from Ares I-X RBM testing, steady and unsteady floor rail pressure transducers were installed for the Ares I RBM test to obtain shock reflection data and to quantify unsteady phenomena associated with the wind-tunnel environment.

The design of the sensor orifice and installation geometry must not alter the local airflow phenomena being measured. Furthermore, the cavity formed by the orifice depth and the sensor head beneath the orifice must possess a resonant cavity frequency well above (preferably 5 times) the frequency content of the flow field to be measured. Figure 7 illustrates the installation approach used in the Ares buffet test program for the majority of the pressure transducers, which consists of a flare tube nut, washer, and o-ring. This mount design allows for easy removal and recovery of Kulites, but proved troublesome for a small fraction $(\sim 10 \%)$ of sensor mountings which exhibited moderate pressure leaks. It was determined through frequency response testing that these moderate leaks did not affect the sensor response in the measured frequency range. Due to limited internal volume available in some sections of the model such as the LAS, 072-size Kulites were used and were bonded into a 0.081 inch diameter hole using adhesive. For aeroacoustic bandwidth transducers, the 072-size Kulites are bonded into inserts that are mounted within the body of the model (Figure 8). The face of the insert is then hand-worked to match the outer mold line of the model.

Table 3 lists the installation geometry dimensions for the Kulite mount design presented in Figure 9. Estimates of cavity resonance using formula for Helmholtz resonators found in Reference 15 for each transducer installation are presented in Table 4 along with pertinent cavity resonance parameters. The back-side reference tube of each model Kulite was attached to manifolds which referenced all Kulites to the plenum static pressure of the TDT. Alternately, a solenoid valve was employed to route a calibration pressure to all Kulite reference line manifolds for in-situ sensor calibration and health monitoring.

\section{Data Acquisition Systems}

Three independent data acquisition systems were employed at the TDT to acquire test data in support of the objectives of the Ares RBM test program. The first system, known as the Open Architecture Data Acquisition System (OADAS) and based on NEFF 620 hardware, was used to acquire low bandwidth (up to $200 \mathrm{~Hz}$ ) data such as tunnel conditions, model orientation data, model vibration response accelerometer data, and floor and ceiling rail unsteady and steady pressure data (Ares I RBM only). The second data system acquired buffet pressure data and is known as the Multi-Instrument Integrated Data Acquisition System (MIIDAS) and is based on NEFF 730 hardware. The MIIDAS system acquired all 256 RBM unsteady pressure measurements and model vibration response accelerometer data at a sample rate of $12 \mathrm{KHz}$ ( $4500 \mathrm{~Hz}$ bandwidth). The third data system used was the Piranha III Digital Dynamic Data Monitoring and Analysis System developed by DSPCon. It acquired secondary test objective aeroacoustic bandwidth data on the Ares I RBM at a sample rate of $100 \mathrm{KHz}$.

\section{Test Implementation}

\section{A. Instrumentation}

Following installation of the model and instrumentation, checkout of model systems and facility data systems commenced to verify data system channel identification and that all transducers and associated reference tubing were functional. Checkout testing included: front-side port leak checks, back-side reference tube and manifold leak checks, channel/transducer identification, transducer calibration, and verification of calibration constants by applying a known front-side port pressure. Pressure transducer calibrations were carried out using a Pressure Systems Inc. ESP system Pressure Calibration Unit to apply 10 or more steady pressures to the back-side reference tube of each pressure transducer and measuring the subsequent response voltages by the OADAS, MIIDAS, and Piranha III. An appropriate curve fit was then applied to the calibration data to obtain engineering unit conversion constants.

The frequency response of each Kulite pressure transducer was confirmed in-situ by applying white noise sound pressure to each model pressure port using a speaker driver with specialized test cap with 0.125 inch reference microphone (Figures 10 and 11). The microphone and Kulite sensor signals were acquired simultaneously to allow for a frequency response function to be computed for all 256 model Kulites. Figure 12 presents representative buffet 
and aeroacoustic bandwidth Kulite frequency response functions measured in-situ within the Ares I RBM. Frequency response is shown to be within $\pm 1 \%$ at $1000 \mathrm{~Hz}$ model-scale and phase shift of less than $5^{\circ}$.

\section{B. Test Conditions and Procedures}

Buffet testing of the Ares I and I-X RBMs was focused on the transonic regime and was conducted at Mach numbers of $0.50,0.70,0.80,0.82,0.84,0.85,0.86,0.88,0.90,0.92,0.94,0.95,0.96,0.98,1.00,1.05,1.10,1.15$, and 1.20. Dynamic pressure was typically the highest attainable at the test facility, with limited testing conducted at lower dynamic pressures to determine Reynolds number effects on buffet pressures. Table 1 lists TDT test section flow conditions (Reynolds number based on model first stage diameter) for typical RBM test matrix conditions and target model-scale bandwidth requirements. Model pitch orientation was set from -8 to +8 deg in 1 degree increments and model roll orientation was set from -180 to +180 deg in 15 degree increments. The sensitivity of buffet loads to vehicle protuberances was determined via testing of both the clean (no protuberances) and the protuberance-on configurations.

Once steady flow conditions were obtained in the test section, thirty second time history records were simultaneously acquired using the OADAS and MIIDAS data systems for buffet bandwidth objectives. Ten second records were acquired using the DSPCon data system for aeroacoustic test objectives. Shadowgraphs of the transonic/supersonic flow-field were acquired using a high-intensity light source and video cameras to aid in the identification of flow phenomena.

\section{Data Analysis}

The primary products of the Ares I RBM test program are full-scale buffet forcing functions that consist of time histories of orthogonal centerline loads $\left(F_{x}, F_{y}\right.$, and $\left.F_{z}\right)$ due to fluctuating buffet pressures along the length of the vehicle. These loads are computed by integrating pressures at each model station possessing a ring of transducers. Analysis of RBM data also includes the scaling of model scale results to full scale values, calculation of power spectral densities of pressure coefficients and BFF results, and one-third octave aeroacoustic results. Ultimately, the time domain BFFs are applied to a finite element model of the CLV as part of a time domain buffet loads analysis (ref. 6). After combining the resulting buffet loads with loads from other launch vehicle disciplines, the total loads, stresses, and accelerations on the full scale vehicle are assessed by coupled loads analysis (CLA) techniques. Buffet loads analysis and CLA are not addressed within this paper.

\section{A. Scaling Laws}

Model scale time histories of pressure and force must be scaled to full scale quantities in order to be included in vehicle load analyses using the scaling laws listed in Table 2. Full-scale launch vehicle trajectory conditions (Mach, $\mathrm{Q}, \mathrm{V}$, etc. versus time) are determined by interpolation using the model-scale Mach number. These interpolated fullscale flight conditions are then used to scale the magnitude and time-scale (sample rate) of the measured windtunnel RBM buffet environment.

\section{B. Forcing Function Development}

Buffet forcing functions (BFFs) are a series of orthogonal force time histories acting at the centerline of a launch vehicle (See Figure 13). These time histories represent the unsteady aerodynamic loads acting on each segment of the model due to the buffet environment. The loads are obtained by integrating measured pressures from rings of transducers distributed along the longitudinal axis of the model. The buffet forcing functions can be calculated using two methods of pressure integration yielding either sectional (force/length) or point (force) loads; each useful for different types of analyses and comparisons. A sectional load, depicted in

Figure 14a, is calculated by integrating the measured pressures at a station along the circumference at that station to produce a set of orthogonal loads acting at the centerline of the segment. These loads have units of force-perlength and are useful for comparing the relative magnitude of the buffet loads at various vehicle stations, independent of sensor distribution. Point forces, portrayed in

Figure 14b, are calculated by integrating the measured pressures circumferentially and longitudinally over a segment of the model. Loads calculated employing this method are used to conduct the buffet loads analysis. Comparison results of the buffet environments found in this paper will employ non-dimensionalized sectional buffet forcing function root-mean-squared (rms) results.

\section{Reducing Over-Conservatism in Buffet Forcing Functions}


The assumption that measured pressure fluctuations over each transducer's defined zone of integration are fully correlated can result in buffet forcing functions that are too conservative. This over-conservatism arises because the integration process assumes that, at any instant in time, the measured pressure is applied to the entire area of integration. In reality, the magnitude and phase of the fluctuation pressures change as the fluid moved down the vehicle and therefore the pressures do not act in unison across the integration area. The over-conservatism can be amplified by increasing transducer separation, which increases the longitudinal length of integration zones over which measured pressures are integrated (and assumed fully correlated over that zonal area). The level of correlation between two transducer measurements can be quantified by calculating the coherence between the two signals. The coherence between two transducer measurements is a function of frequency, and the coherence values can range between zero and one. The coherence decreases exponentially with increasing transducer separation distance, signaling that the measured pressure time histories are becoming less correlated. This observation is important in buffet load analysis because the more correlated the transducer signals are, the greater the effective loading. In other words, as the transducer spacing increases and the coherence decreases, it becomes more inaccurate to integrate the measured pressures over the entire transducer zonal area. Therefore, a coherence based method of adjusting the integration area is used to avoid over-conservatism in the integrated BFFs.

A technique based on longitudinal coherence is employed in the present analysis to reduce this overconservatism, thereby avoiding unrealistically high buffet loads and thus reducing vehicle structural margins. Azimuthal coherence and reduction of the azimuthal integration arc length were not considered because some degree of conservatism was desired. The method was developed based on a technique used by Aerospace Corporation in their buffet analysis of previous launch vehicles. This method for reducing over-conservatism in the buffet forcing functions is a multi-step process involving the identification of regions based on flow characteristics, estimation of flow coherence, calculation of the knockdown factors, and the application of these knockdown factors to the BFFs.

\section{Notch Filtering of Facility Blade Passage Frequencies}

Harmonics of blade passage frequencies (BPFs) due to the TDT main drive fan are present in measured RBM BFF time histories. The variation with Mach number of the fundamental blade passage frequency due to drive fan speed is presented in Table 5 for model-scale frequencies. The first three harmonics of blade passage frequency within the buffet forcing functions are considered significant and are not present in the Ares I buffet environment, but rather an artifact of the wind-tunnel test environment. The presence of these BPFs could potentially couple with vehicle structural modes when buffet forcing functions are applied to an analytical structural model during coupled loads analysis and lead to erroneous vehicle loads. Thus, a method for attenuating the BPFs within the pressure forcing functions and buffet forcing functions has been implemented.

Since the magnitude of each BPF harmonic varies within each forcing function, the filter design must adapt to these varying magnitudes. The approach implemented follows that put forward by the Aerospace Corporation and involves using Principal Component Analysis (PCA) and Singular Value Decomposition (SVD) to smooth the PSDs of forcing functions in the vicinity of the BPF harmonics to provide accurate identification of the noise floor at BPF harmonics. A unique filter Frequency Response Function (FRF) is designed for each forcing function to account for variability of the magnitudes of the BPFs and the need for unique attenuation of BPFs. Each filter FRF is applied to the proper buffet forcing function using an FFT filter which is a type of finite impulse response, or FIR, filter. Figure 15 compares the PSDs of an unfiltered BFF and one that has been notch filtered to remove the blade passage frequencies. As shown, the filter design is such that only the frequency content in the vicinity of the blade passage frequency is attenuated to a level consistent with the local noise floor. Figure 16 shows the FRF between the filtered and unfiltered buffet forcing function for station FY18 which demonstrates that the filter design does not significantly affect the filtered signal magnitude and phase (time-correlation) outside of the BPF notch filter bands.

\section{Buffet Environment Results}

Buffet environment results in this section focus on steady and root-mean-square values and time histories of fluctuating buffet pressures and buffet forcing functions across the Mach number range tested. Trends in buffet forcing functions with respect to Mach number $(M)$ and model pitch angle $(\theta)$ are presented within plots of fluctuating root-mean-squared components of lateral and vertical loads. All buffet forcing function root-meansquare magnitudes are computed from time histories that have been bandpass filtered between $0.5 \mathrm{~Hz}$ and $60 \mathrm{~Hz}$ (full-scale) in order to focus analysis on frequency content that will most affect the buffet loads on the vehicle.

\section{A. Peak Fluctuating Buffet Pressures}


Fluctuating pressure levels have been typically reported in past launch vehicle literature by presenting the rootmean-squared value of the fluctuating component of pressure from static conditions (refs. 1-3). The rms value of the fluctuating pressure coefficient, $\Delta C_{p, r m s}$, indicates the degree of fluctuating buffet pressures due to shock oscillations, Strouhal shedding, and turbulence phenomena, and where this value is largest would indicate areas prone to high buffet loads on a launch vehicle. Figures 17 and 18 present contour plots of peak $\Delta C_{p, r m s}$ versus model pitch angle and Mach number for a R134a test medium with all vehicle protuberances installed. The regions of peak $\Delta C_{p, r m s}$ are quite different for the two vehicles. For the Ares I-X contour plot in Figure 17, the regions of peak $\Delta C_{p, r m s}$ are dominated by a flow environment known as alternating flow separation and attachment (AFS\&A) due to the sharp corner of the cone-cylinder junction at the command module (CM) and service module (SM) interface ${ }^{16}$. Regions of peak $\Delta C_{p, r m s}$ for the Ares I RBM contour plot shown in Figure 18 are dominated by terminal shock oscillations on the service module for Mach numbers between 0.8 and 0.90 , shock oscillation due to flow expansion at the frustum centered near Mach 0.98 , and again due to shock oscillation at the frustum in vicinity of Mach $1.10^{17}$.

\section{B. Buffet Forcing Function Trends: Ares I-X}

Ares I-X RBM normalized sectional buffet forcing function rms levels $\left(\Delta C_{y, r m s}\right.$ and $\left.\Delta C_{z, r m s}\right)$ versus longitudinal station for the Ares I-X RBM are presented in Figure 19 for Mach numbers between 0.8 and 1.2 in an R-134a test medium and all vehicle protuberances on. Peak $\Delta C_{y, r m s}$ and $\Delta C_{z, r m s}$ for the Ares I-X configuration can be attributed to the alternating flow separation and attachment phenomena which occurs at a Mach number of 0.9 at the CM/SM cone-cylinder interface. This alternating flow condition is typified by the square-wave like signature of the pressure time histories shown in Figure 20 which results in buffet forcing functions with a pulse-like signature due to azimuthal asymmetry in the flow. Figure 21 presents shadowgraph images of the two flow conditions which randomly and rapidly alternate at this Mach number: (a) subsonic detached flow and (b) supersonic attached flow. The severity of this alternating flow phenomenon demonstrated by the square-wake signature in Figure 20 is exacerbated by the steady flow conditions of the wind-tunnel-test environment. In reality, a launch vehicle which is accelerating constantly within its trajectory would experience a single impulsive load as it passes through the narrow Mach range in which the alternating flow condition exists. Reference 18 presents a more detailed analysis of the alternating flow separation and attachment phenomenon.

Figure 22 and Figure 23 present normalized sectional buffet forcing function rms levels for Mach numbers of 0.9 and 1.2, respectively, for vehicle pitch angles $(\theta)$ from $-4^{\circ}$ to $+4^{\circ}$. Significant variability with BFF rms levels for the transonic Mach 0.9 case (alternating flow) presented in Figure 22 is seen in the vicinity of the service module and spacecraft adapter.

\section{Buffet Forcing Function Trends: Ares I}

Figure 24 and Figure 25 presents $\Delta C_{y, r m s}$ and $\Delta C_{z, r m s}$ versus longitudinal vehicle station for the Ares I RBM for Mach numbers between 0.80 and 1.20 in an R-134a test medium and all vehicle protuberances on. The peak buffet environment for the Ares I vehicle is dominated by terminal shock oscillation on the service module at Mach 0.82 and shock oscillation at the frustum. The Ares I RBM terminal shock oscillation event in vicinity of the service module is seen to result in a higher normalized sectional buffet forcing function rms level than the Ares I-X alternating flow phenomenon (Figure 19 though Figure 25 have identical y-axes limits). However, the terminal shock oscillation observed in tests of the Ares I RBM did not result in square-wave like pressure signatures and was instead a more random noise, broad-band event. The less benign transonic buffet environment (lack of alternating flow condition) of Ares I is attributed to the ogive shape of the command module shroud.

Figure 26 and Figure 27 present Ares I RBM normalized sectional buffet forcing function rms levels for Mach numbers of 0.82 and 1.2, respectively, for vehicle pitch angles $(\theta)$ from $-6^{\circ}$ to $+6^{\circ}$. Significant variability with BFF rms levels for the transonic Mach 0.82 case (oscillating terminal shock) presented in Figure 26 is seen in the vicinity of the service module and spacecraft adapter. Very little sensitivity in vehicle pitch to the buffet forcing function rms levels are shown in Figure 27 for the Mach 1.2 supersonic condition.

\section{Conclusions}

The development of the Ares I-X and Ares I rigid buffet model test program has been presented. The program consisted of tests of two 3.5-percent scale models which were conducted at the NASA Langley Transonic Dynamics Tunnel. The models were instrumented with an array of unsteady pressure transducers in order to acquire unsteady pressure time histories at transonic conditions for frequencies up to $4.5 \mathrm{KHz}$ for buffet data and $100 \mathrm{KHz}$ for aeroacoustic data. The design of the models, their instrumentation, and testing procedures have been discussed. 
The development of buffet forcing functions (BFFs) based on the measured surface pressures has been presented. The procedures outlined include integrating pressures over the surface of the models, development of knockdown factors, filtering, and scaling laws for transforming model-scale BFFs to full-scale.

The peak buffet environments of the Ares I- and I-X vehicles have been compared through the presentation of buffet forcing function root-mean-squared levels along the vehicle centerlines. The most severe Ares I-X buffet loads are a result of an alternating flow attachment and separation phenomenon that can occur at transonic flight conditions. The maximum Ares I buffet loads are caused by terminal shocks oscillating on the ogive fairing and frustum of the upper stage.

\section{Acknowledgments}

The authors would like to acknowledge their collaboration with Jamie Canino, Shin Chen, Sam Lee, and Brian Sako of the Aerospace Corporation on aspects of the buffet forcing function development.

\section{References}

1. Jones, George W., Foughner, Jerome T., Jr., "Investigation of Buffet Pressures on Models of Large Manned Launch Vehicle Configurations”. NASA TN-D-1663, May 1963.

2. Rainey, Gerald A., "Progress on the Launch-Vehicle Buffeting Problem”. Journal of Spacecraft and Rockets, Volume 2, Number 3, May-June 1965.

3. Cole, Henry A., Jr.; Erickson, A. L.; and Rainey, A. G.: "Buffeting During Atmospheric Ascent”. NASA SP8001, 1963, Revised November 1970.

4. Fleming, E.R., "Transonic Buffeting Loads Experience at The Aerospace Corporation”. The Aerospace Corporation Report No. TOR-95(5530)-6. March 1995.

5. Schutzenhofer, Luke A., Howard, Paul W.: “Acquisition and Reduction of Large Volumes of Fluctuating Data”. Aerophysics Research at MSFC: Research Achievements Review. Volume III, Report No. 4. NASA TM X53799, 1968.

6. Kabe, A.M., "Time Domain Buffet Loads Analysis". The Aerospace Corporation Report Number TOR2007(2209)-6078. November 17, 2006.

7. Test Summary Document for the 3.5 Percent Ares I-X Rigid Buffet Model. ARES-AE-TA-0002, Version 1.0, July 11, 2008.

8. Test Summary Document for the 3.5 Percent Ares I Rigid Buffet Model. ARES-AE-TA-0012, Version 1.0, July 14, 2009.

9. Data Analysis and Results Document for the 3.5 Percent Ares I Rigid Buffet Model. ARES-AE-TA-0013, Version 1.0, August 3, 2009.

10. Ares I-X 3.5\% Rigid Buffet Model Full-Scale Buffet Forcing Functions. ARES-AE-TA-0005, Release August 21, 2008.

11. Database of Ares I Full-Scale Buffet Forcing Functions. ARES-AE-DBR-0001, Release 1.1, October 9, 2009.

12. Cole, Stanley R.; Keller, Donald F.; and Piatak, David J.: "Contributions of the NASA Langley Transonic Dynamics Tunnel to Launch Vehicle and Spacecraft Development”. AIAA 2000-1772, Presented at the AIAA Dynamics Specialists Conference, Atlanta, GA, April 5-6, 2000.

13. Cole, Stanley R., Garcia, Jerry L.: "Past, Present, and Future Capabilities of the Transonic Dynamics Tunnel from and Aeroelasticity Perspective”. AIAA 2000-1767, Presented at the AIAA Dynamics Specialists Conference, Atlanta, GA, April 506, 2000. 
14. Cole, Stanley R.; Johnson, R. Keith; Piatak, David J.; Florance, Jennifer P.; and Rivera, José A., Jr.: “Test Activities in the Transonic Dynamics Tunnel and a Summary of Recent Facility Improvements”. AIAA 20031958, Presented at the AIAA Dynamics Specialists Conference, Norfolk, VA, April 9-10, 2003.

15. Blevins, Robert D., “Flow Induced Vibration”. Van Nostrand Reinhold Publishers, ISBN $0442206518,1990$.

16. Piatak, D. J., et al, “Test Summary Document for the 3.5 Percent Ares I-X Rigid Buffet Model”. ARES-AE-TA0002, July 2008.

17. Piatak, D. J., et al, "Data Analysis and Results Document for the 3.5 Percent Ares I Rigid Buffet Model”. ARESAE-TA-0013, July 2009.

18. Sekula, Martin K., Piatak, David J., Rausch, Russ D., “Analysis of a Transonic Alternating Flow Phenomenon Observed During Ares Wind Tunnel Tests”. 
Table 1. Ares RBM TDT test section conditions in R134a test medium.

\begin{tabular}{|c|c|c|c|c|c|}
\hline $\begin{array}{c}\text { Mach } \\
\text { Number }\end{array}$ & q, psf & $\begin{array}{c}\mathbf{V}, \\
\mathbf{f t} / \mathbf{s}\end{array}$ & $\mathbf{R e}, \mathbf{f t}$ & $\begin{array}{c}\text { Re, 1st } \\
\text { Stage Dia. }\end{array}$ & $\begin{array}{c}\text { Model Scale } \\
\text { Buffet } \\
\text { Bandwidth, Hz } \\
\text { (Aeroacoustic) }\end{array}$ \\
\hline \hline 0.5 & 199 & 278 & $5.56 \mathrm{E}+06$ & $2.37 \mathrm{E}+06$ & $\begin{array}{c}0-798 \\
(0-26,597)\end{array}$ \\
\hline 0.8 & 481 & 441 & $8.47 \mathrm{E}+06$ & $3.61 \mathrm{E}+06$ & $\begin{array}{c}0-870 \\
(0-29,000)\end{array}$ \\
\hline 0.9 & 480 & 499 & $7.47 \mathrm{E}+06$ & $3.18 \mathrm{E}+06$ & $\begin{array}{c}0-822 \\
(0-27,410)\end{array}$ \\
\hline 1.0 & 480 & 546 & $6.92 \mathrm{E}+06$ & $2.95 \mathrm{E}+06$ & $\begin{array}{c}0-886 \\
(0-29,519)\end{array}$ \\
\hline 1.2 & 300 & 653 & $3.66 \mathrm{E}+06$ & $1.56 \mathrm{E}+06$ & $\begin{array}{c}0-907 \\
(0-30,238)\end{array}$ \\
\hline
\end{tabular}

Table 2. Rigid buffet model scaling laws.

\begin{tabular}{|c|c|}
\hline $\begin{array}{c}\text { Quantity } \\
\text { to be } \\
\text { Scaled }\end{array}$ & Full scale to Model scale Relationship \\
\hline \hline Pressure & $p_{f s}=p_{m s} \frac{q_{f s}}{q_{m s}}$ \\
\hline Force & $F_{f s}=F_{m s} \frac{q_{f s}}{q_{m s}}\left(\frac{D_{f s}}{D_{m s}}\right)^{2}$ \\
\hline $\begin{array}{c}\text { Time } \\
\text { Frequency }\end{array}$ & $T_{f s}=T_{m s} \frac{D_{f s}}{D_{m s}} \frac{V_{m s}}{V_{f s}}$ \\
\hline $\begin{array}{c}\text { PSD } \\
\left(\text { psi }{ }^{2} / \mathrm{Hz}\right)\end{array}$ & $\Phi_{f s}^{(\text {Pressure })}=\Phi_{m s}^{(\text {Pressure })}\left(\frac{q_{f s}}{q_{m s}}\right)^{2} \frac{D_{f s}}{D_{m s}} \frac{V_{m s}}{D_{f s}}$ \\
\hline $\begin{array}{c}\text { Force } \\
\text { PSD } \\
\left(\text { lbf }{ }^{2} / \mathrm{Hz}\right)\end{array}$ & $\Phi_{f s}^{(\text {Force })}=\Phi_{m s}^{(\text {Force })}$ \\
\hline
\end{tabular}

Table 3. Installation geometry for Kulite pressure transducers.

\begin{tabular}{|c|c|c|c|c|c|}
\hline $\begin{array}{c}\text { Installation } \\
\text { Type }\end{array}$ & $\begin{array}{c}\mathbf{D}_{\text {orifice}} \text {, } \\
\text { in }\end{array}$ & $\begin{array}{c}\mathbf{D}_{\text {sensor, }} \text { in } \\
\text { in }\end{array}$ & $\begin{array}{c}\mathbf{L}_{\text {orifice, }} \\
\text { in }\end{array}$ & $\begin{array}{c}\mathbf{L}_{\text {sensor, }} \\
\text { in }\end{array}$ & $\begin{array}{c}\text { Cavity } \\
\text { Volume, in } \\
\text { (frustum only) }\end{array}$ \\
\hline \hline 093 Buffet & 0.040 & 0.099 & 0.045 & 0.063 & $7.2387 \mathrm{e}-5$ \\
\hline 072 Buffet & 0.040 & 0.081 & 0.040 & 0.052 & $3.5817 \mathrm{e}-5$ \\
\hline 072 Acoustic & 0.040 & 0.081 & 0.010 & $\mathrm{n} / \mathrm{a}$ & 0 \\
\hline
\end{tabular}

Table 4. Cavity resonance parameters and predictions for RBM Kulites.

\begin{tabular}{|c|c|c|c|c|}
\hline $\begin{array}{c}\text { Installation } \\
\text { Type }\end{array}$ & $\mathbf{A}_{\text {orifice, }}$ in $^{2}$ & $\begin{array}{c}\mathbf{V}_{\text {cavity, in }}{ }^{3} \\
\text { [frustum + } \\
\text { sensor] }\end{array}$ & $\begin{array}{c}\mathbf{L}_{\text {Orifice, }} \\
\text { in }\end{array}$ & $\begin{array}{c}\text { Predicted } \\
\text { Cavity } \\
\text { Resonance, } \\
\text { Hz (R134a) }\end{array}$ \\
\hline \hline 093 Buffet & $1.25664 \mathrm{e}-3$ & $2.0039 \mathrm{e}-004$ & 0.045 & 12,239 \\
\hline 072 Buffet & $1.25664 \mathrm{e}-3$ & $9.0817 \mathrm{e}-005$ & 0.040 & 18,588 \\
\hline 072 Acoustic & $1.25664 \mathrm{e}-3$ & $1.4000 \mathrm{e}-005$ & 0.010 & 78,313 \\
\hline
\end{tabular}


Table 5. TDT blade passage frequencies

\begin{tabular}{|c||c|c|c|c|c|c|}
\hline $\begin{array}{c}\text { Mach } \\
\text { Number }\end{array}$ & 0.80 & 0.85 & 0.90 & 0.95 & 1.10 & 1.20 \\
\hline $\begin{array}{c}\text { Motor } \\
\text { Speed } \\
\text { (RPM) }\end{array}$ & 190.8 & 196.6 & 201.5 & 211.4 & 234.4 & 232.4 \\
\hline $\begin{array}{c}\text { Blade } \\
\text { Passage } \\
\text { Frequency } \\
\text { (Hz) }\end{array}$ & 149.5 & 154.0 & 157.8 & 165.6 & 183.6 & 182.0 \\
\hline
\end{tabular}

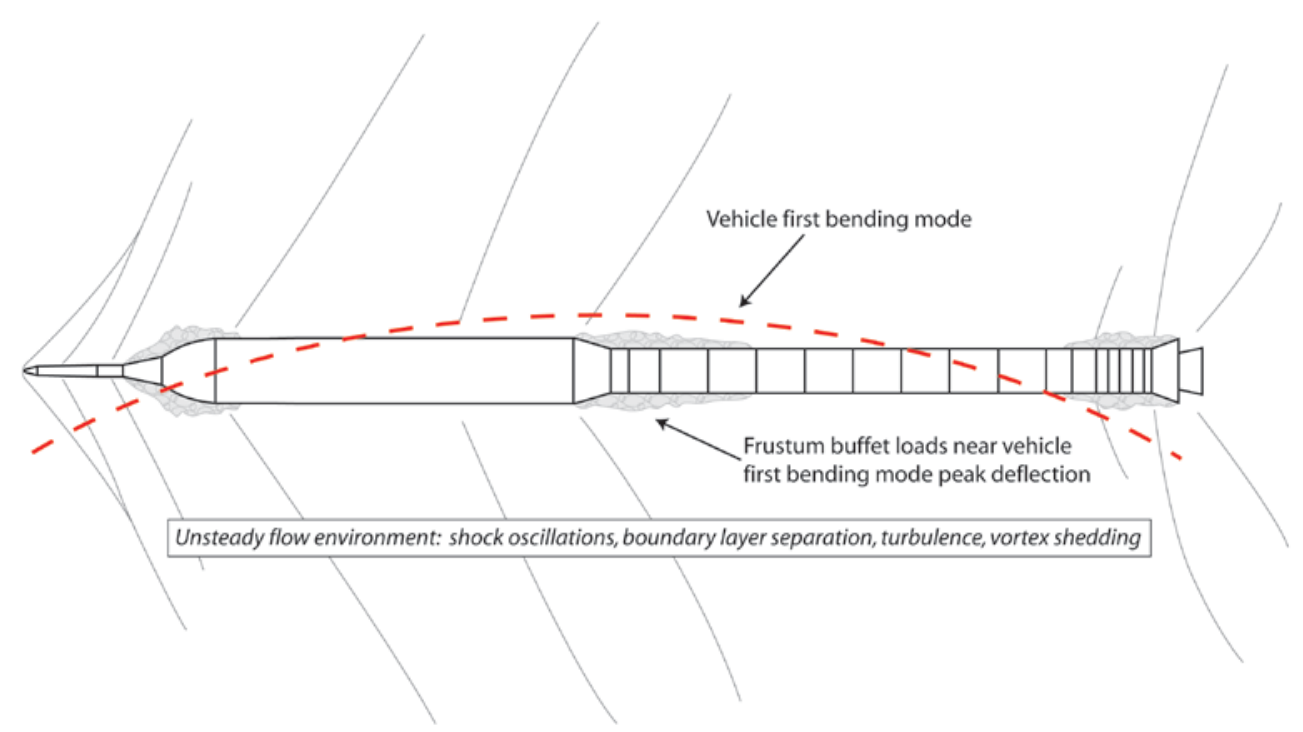

Figure 1. Notional unsteady flow environment for Ares CLV configuration.

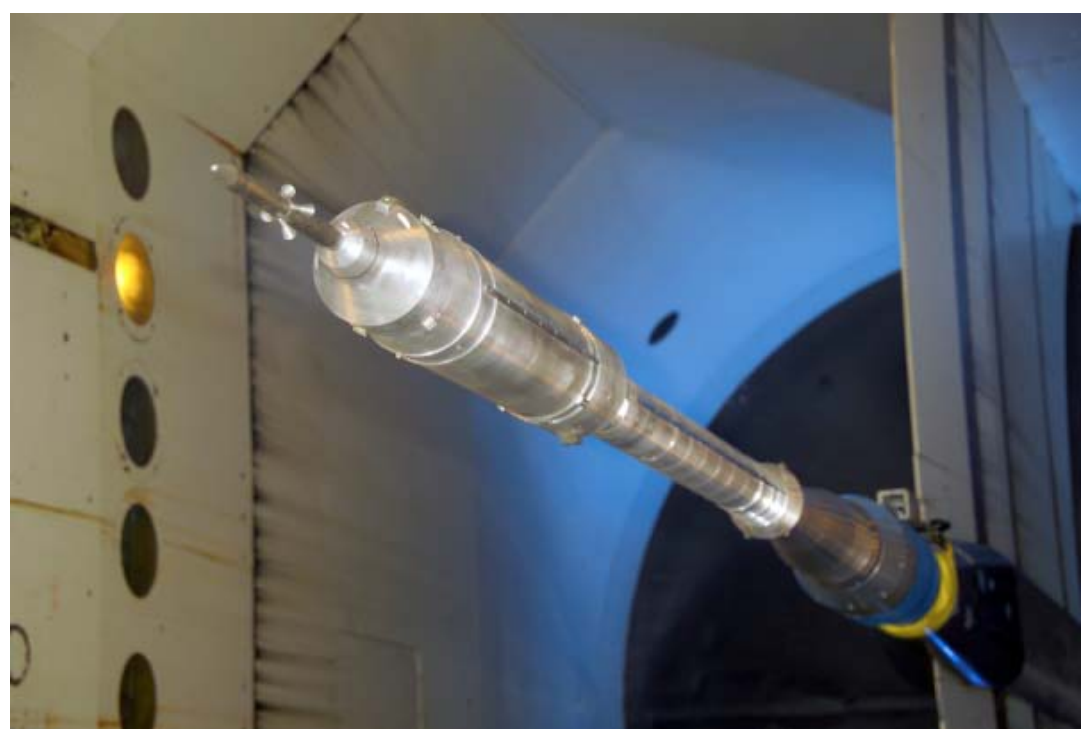

Figure 2. Ares I-X Rigid Buffet Model in TDT (close-up). 


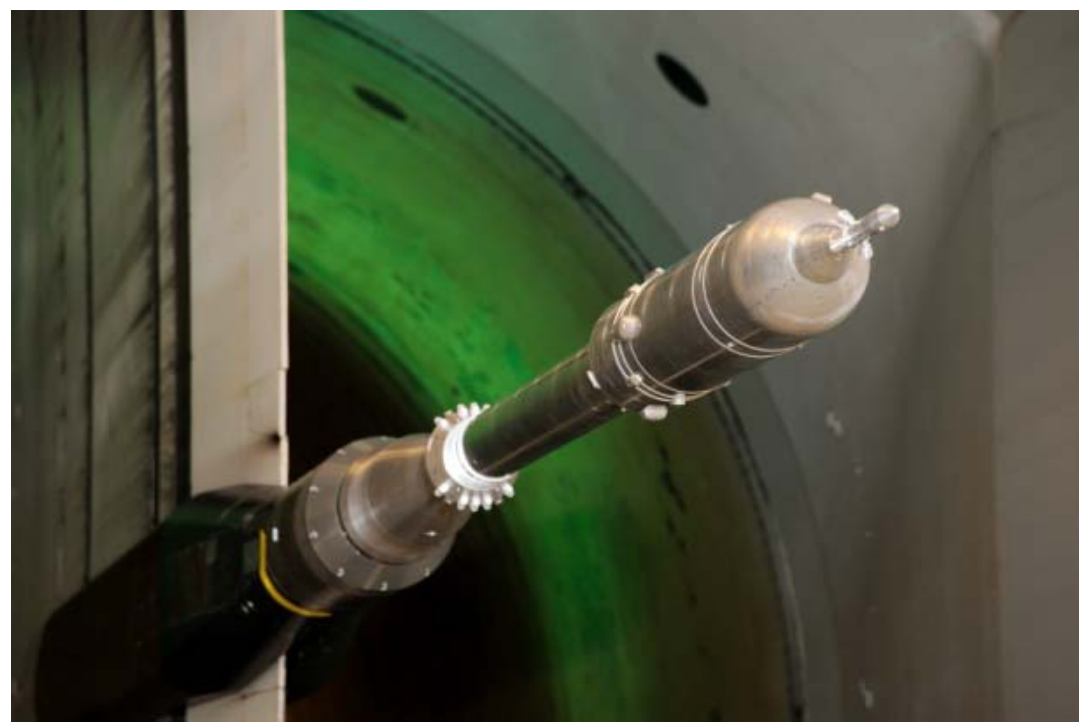

Figure 3. Ares I Rigid Buffet Model in TDT (close-up).

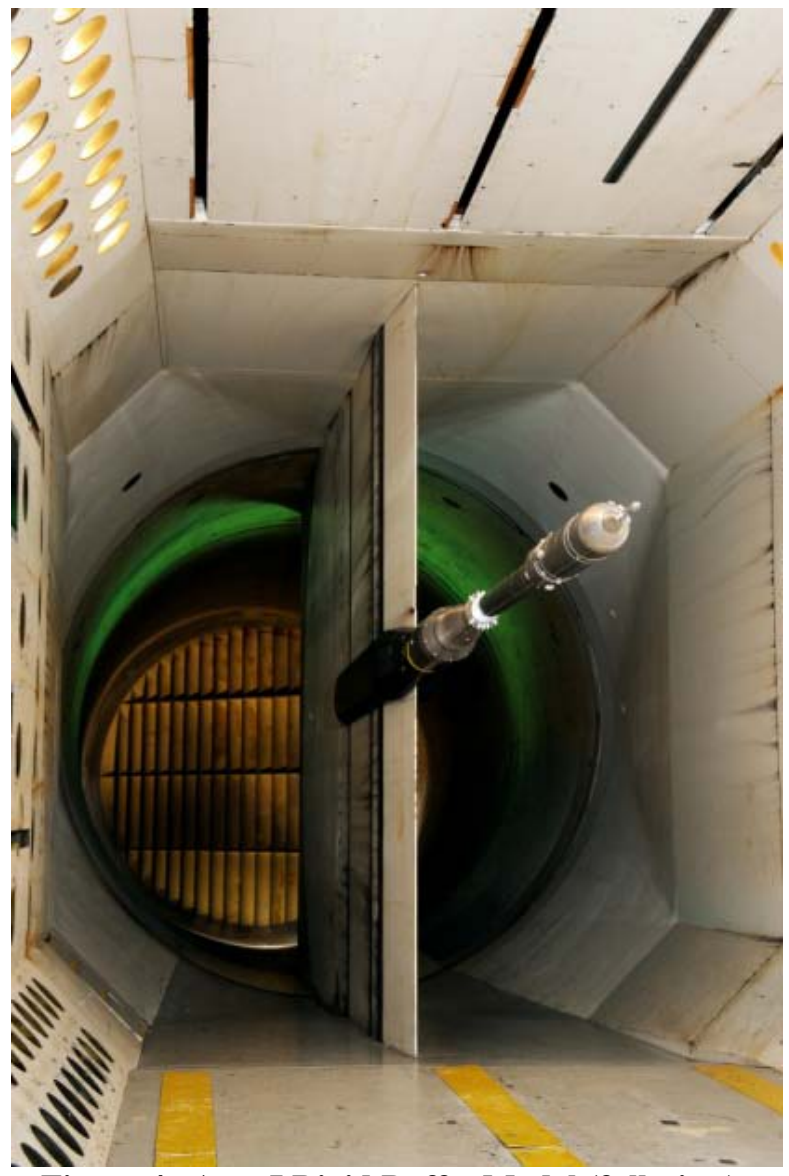

Figure 4. Ares I Rigid Buffet Model (full-view). 


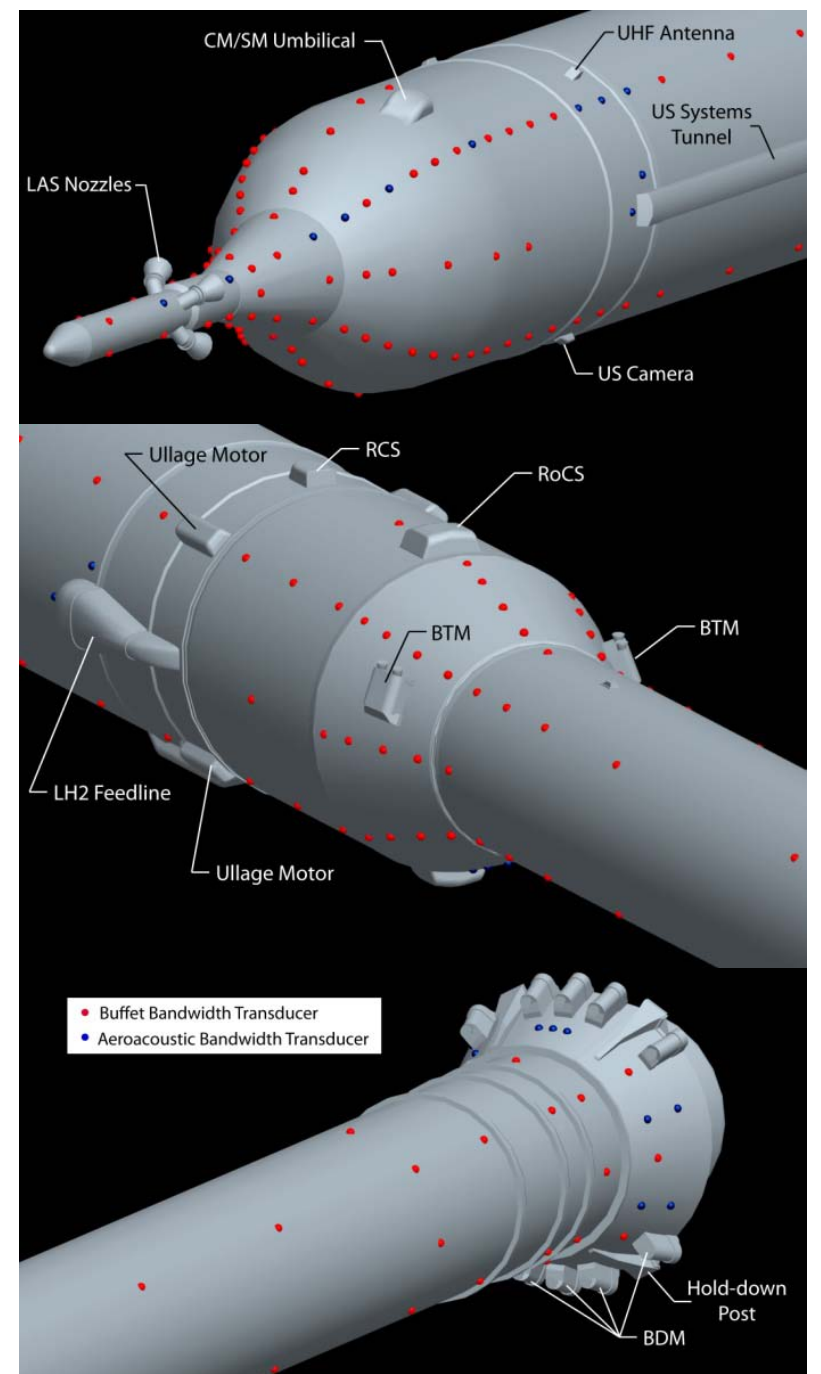

Figure 5. Pressure transducer locations for the Ares I RBM. 


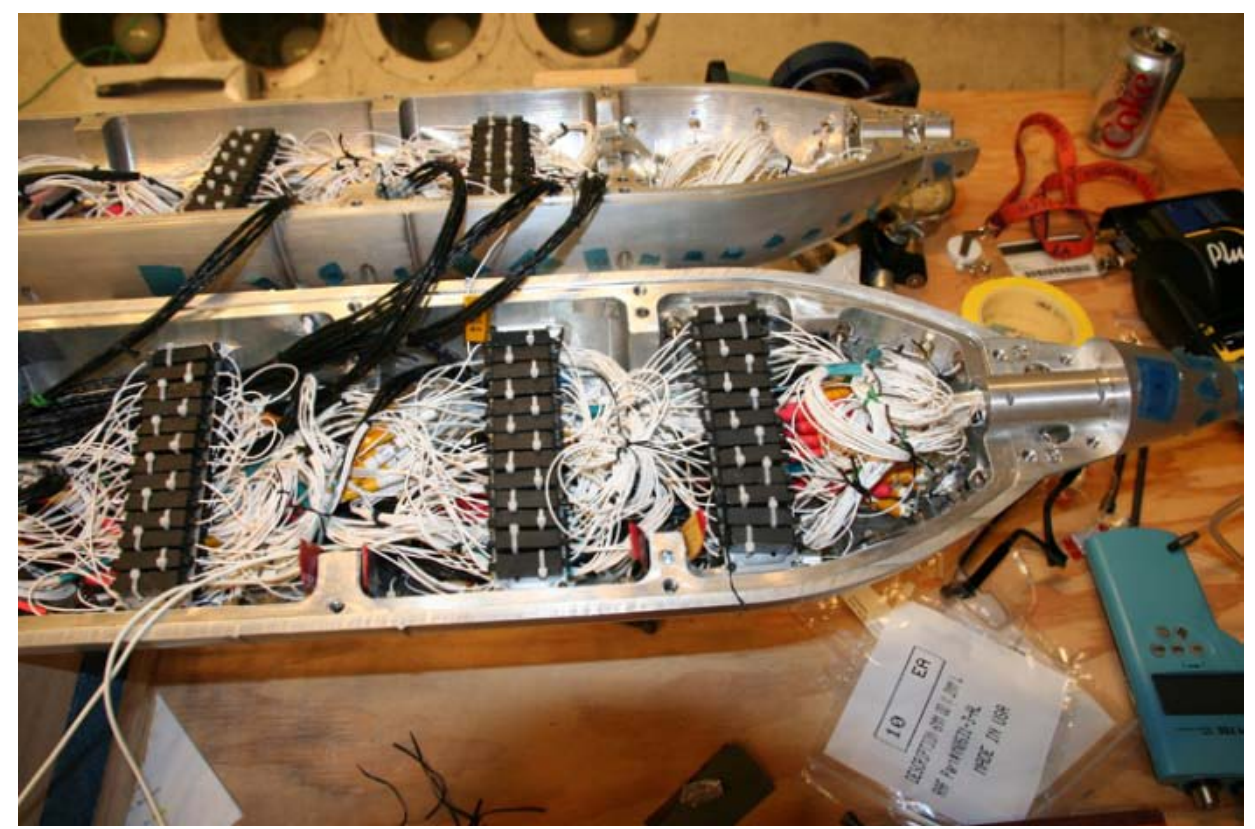

Figure 6. Ares I RBM unsteady pressure instrumentation installation.

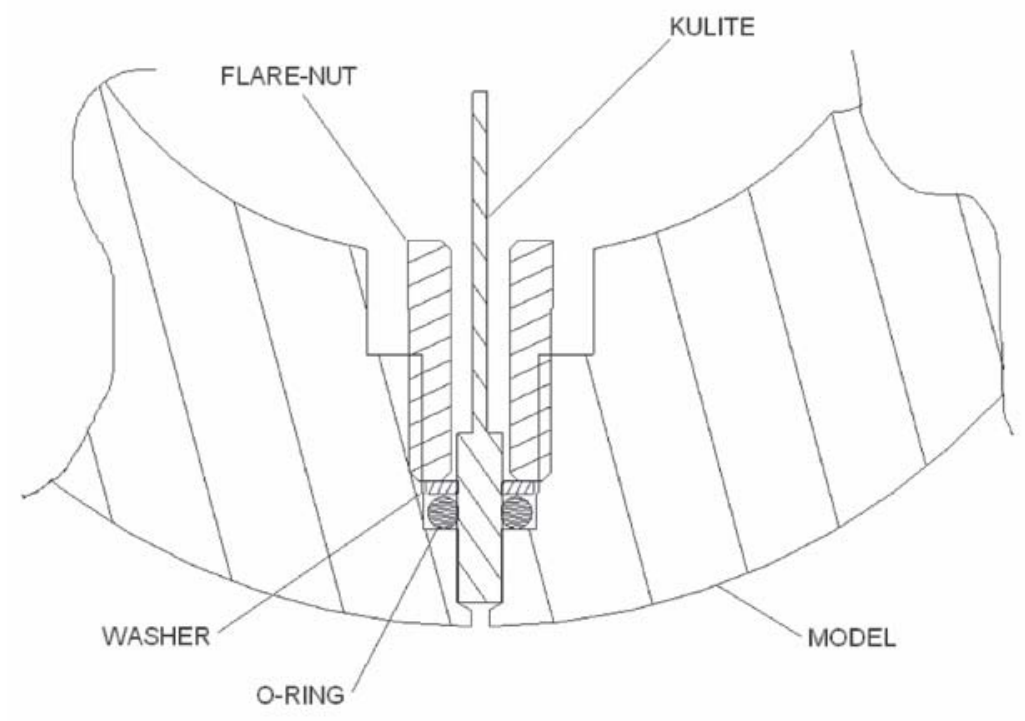

Figure 7. Details of Kulite installation. 


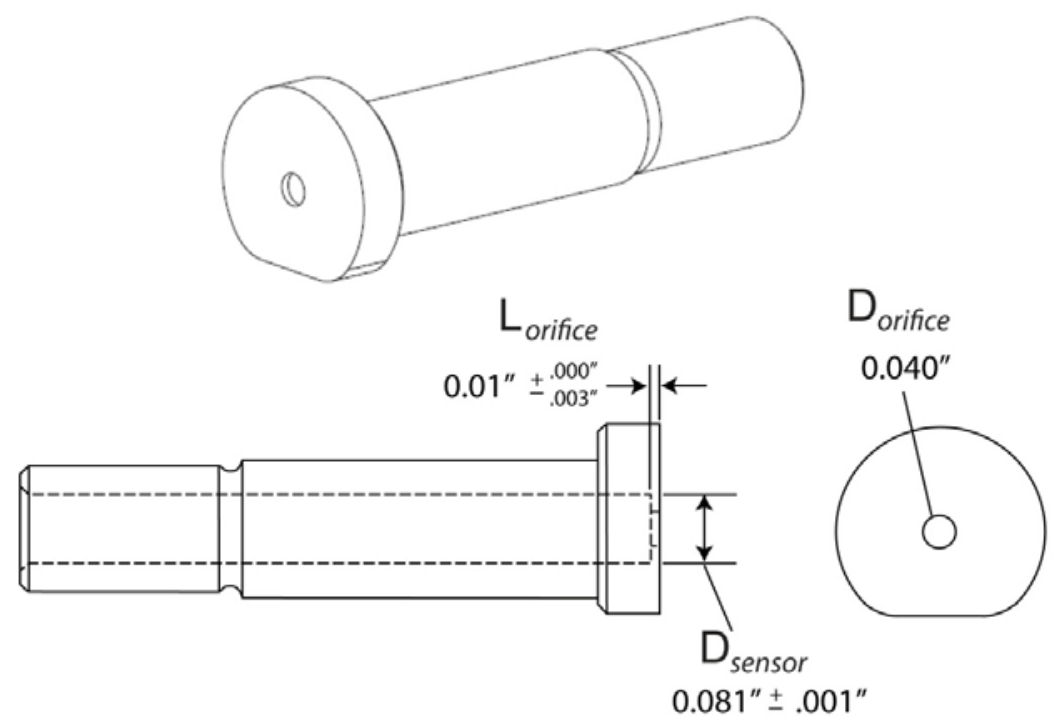

Figure 8. Aeroacoustic insert geometry.

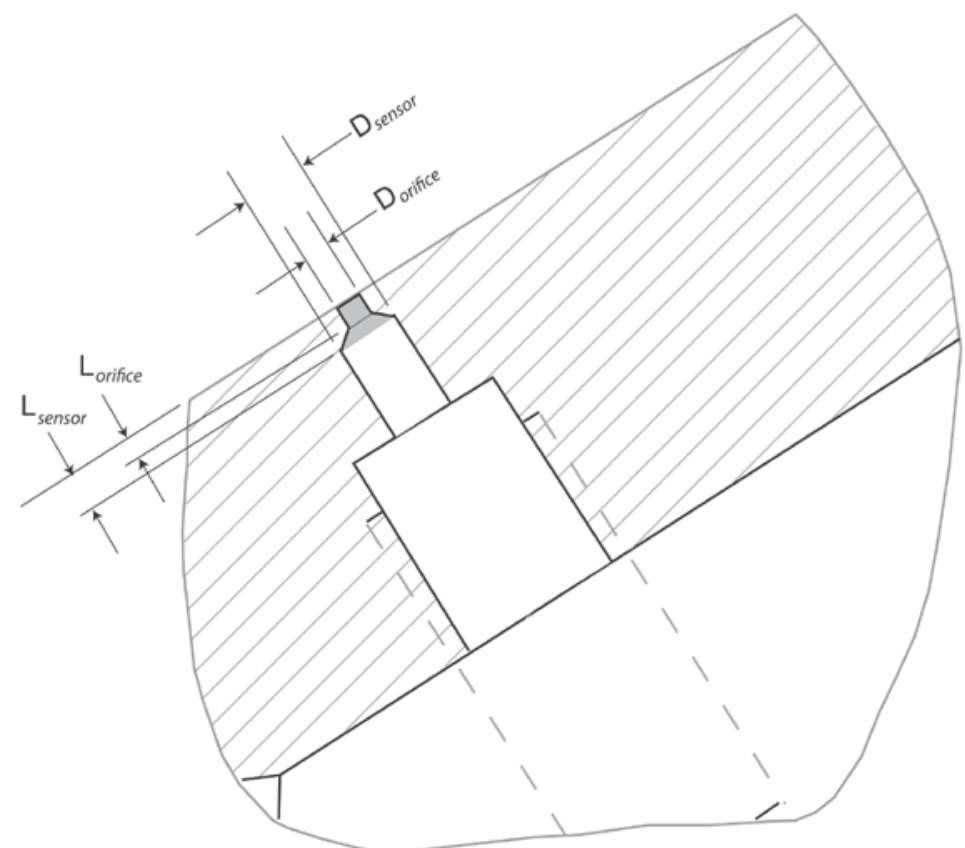

Figure 9. Installation geometry of buffet bandwidth Kulite unsteady pressure transducers. 


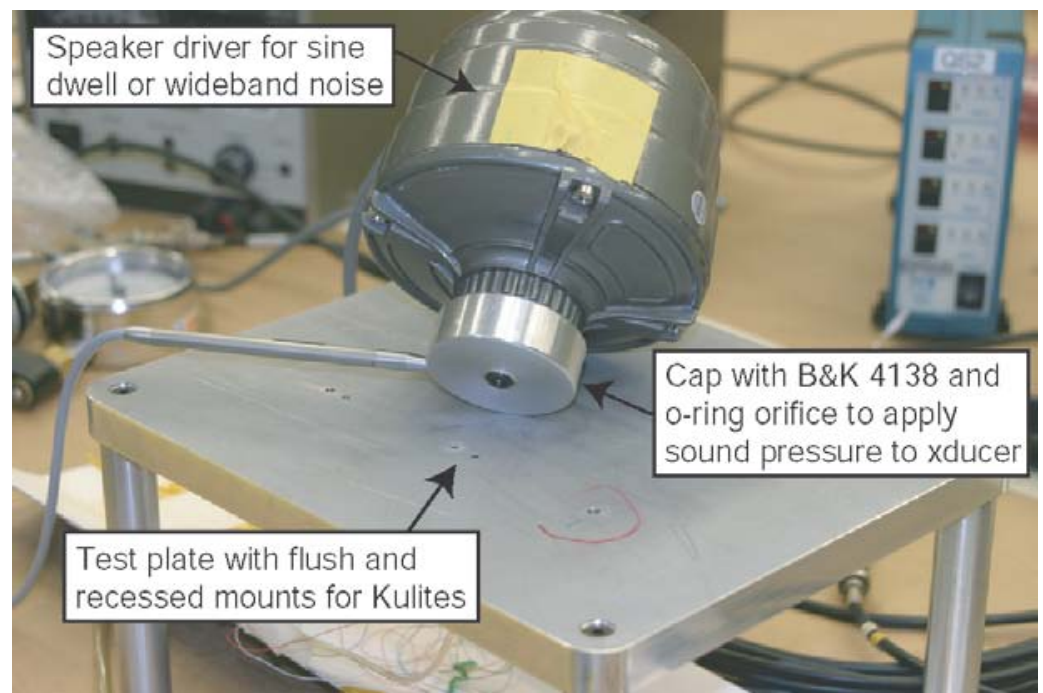

Figure 10. Speaker driver with acoustic test cap for pressure sensor frequency response measurement.

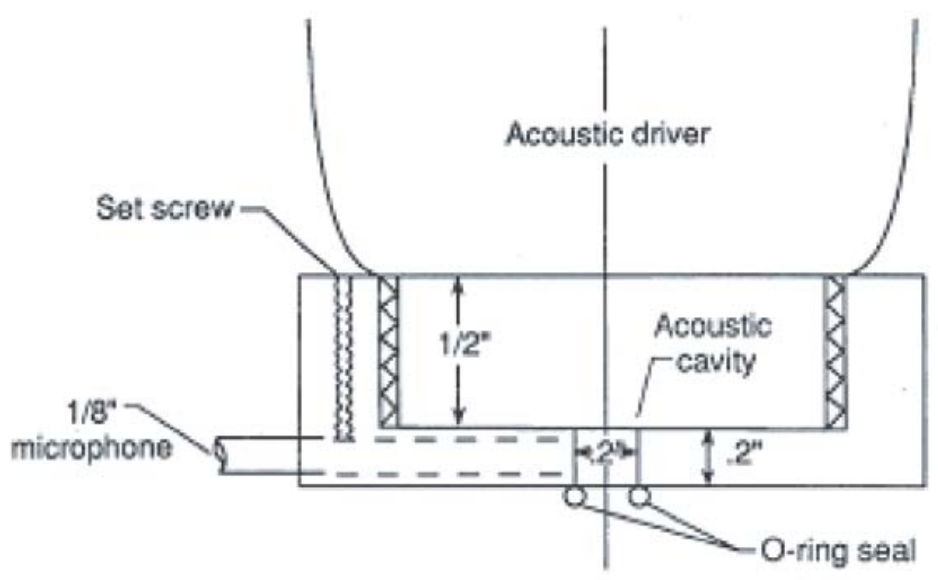

Figure 11. Details of acoustic test cap. 

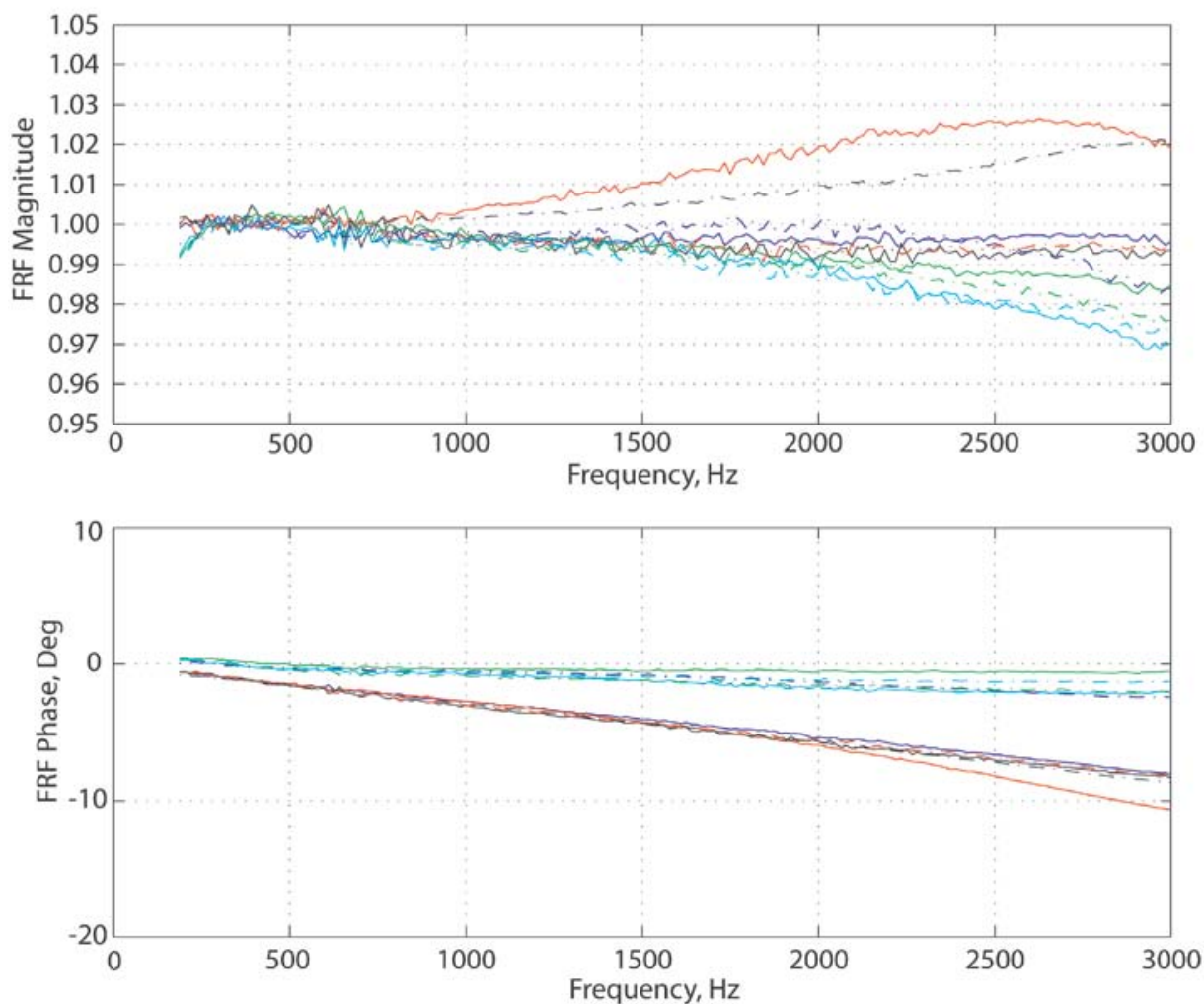

Figure 12. In-situ frequency response functions (FRFs) of representative Ares I RBM buffet and aeroacoustic Kulite pressure transducers (includes effects of instrumentation cabling and signal conditioning).

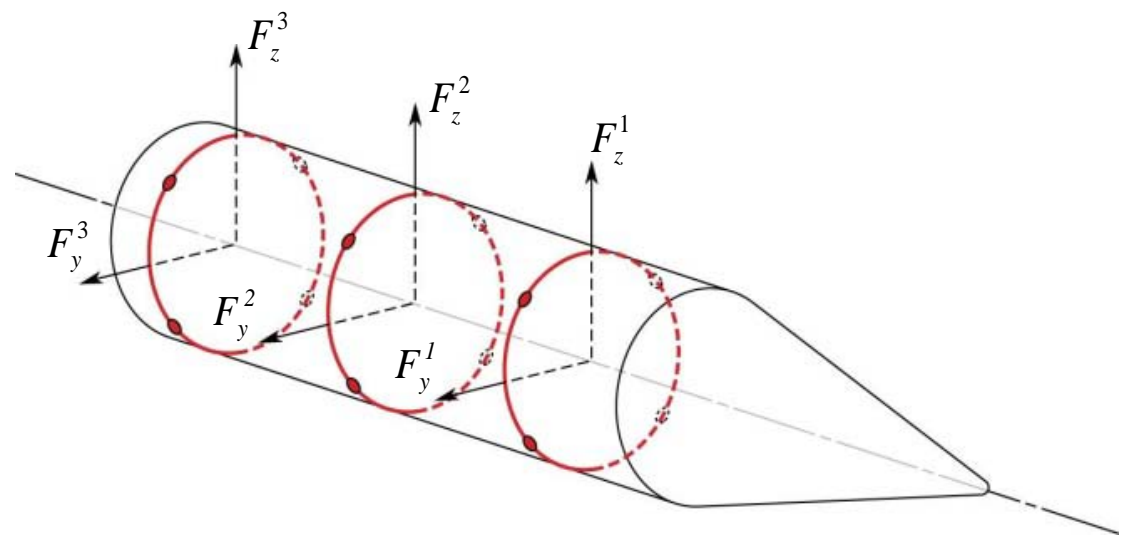

Figure 13. Forcing functions acting on the centerline of vehicle. 


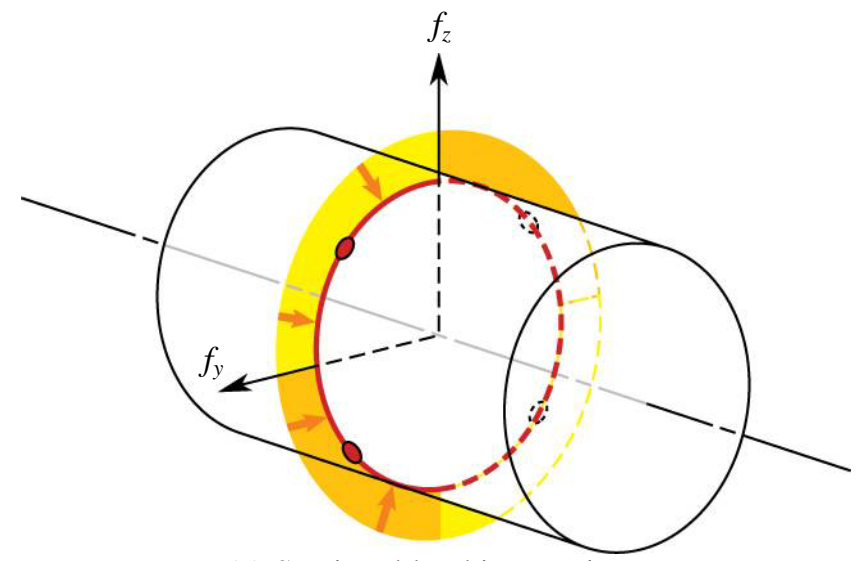

(a) Sectional load integration.

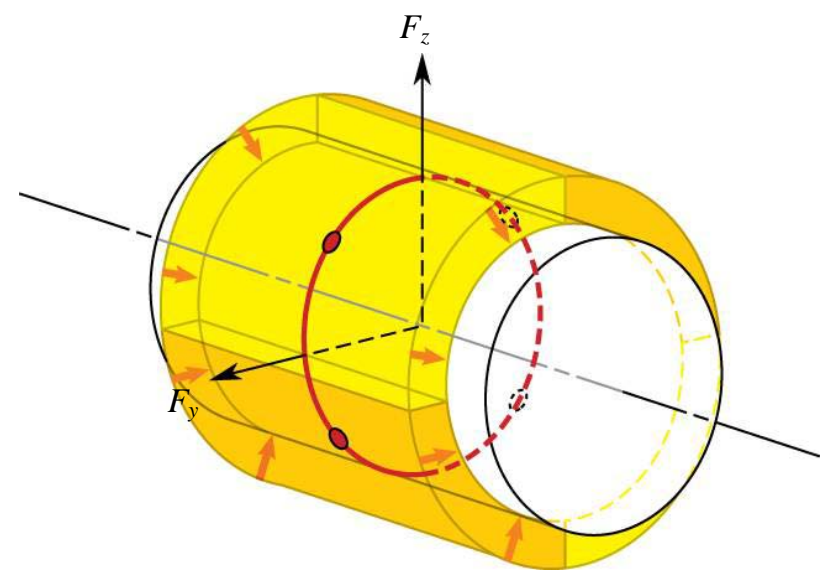

(b) Point load integration

Figure 14. Methods of pressure integration. 


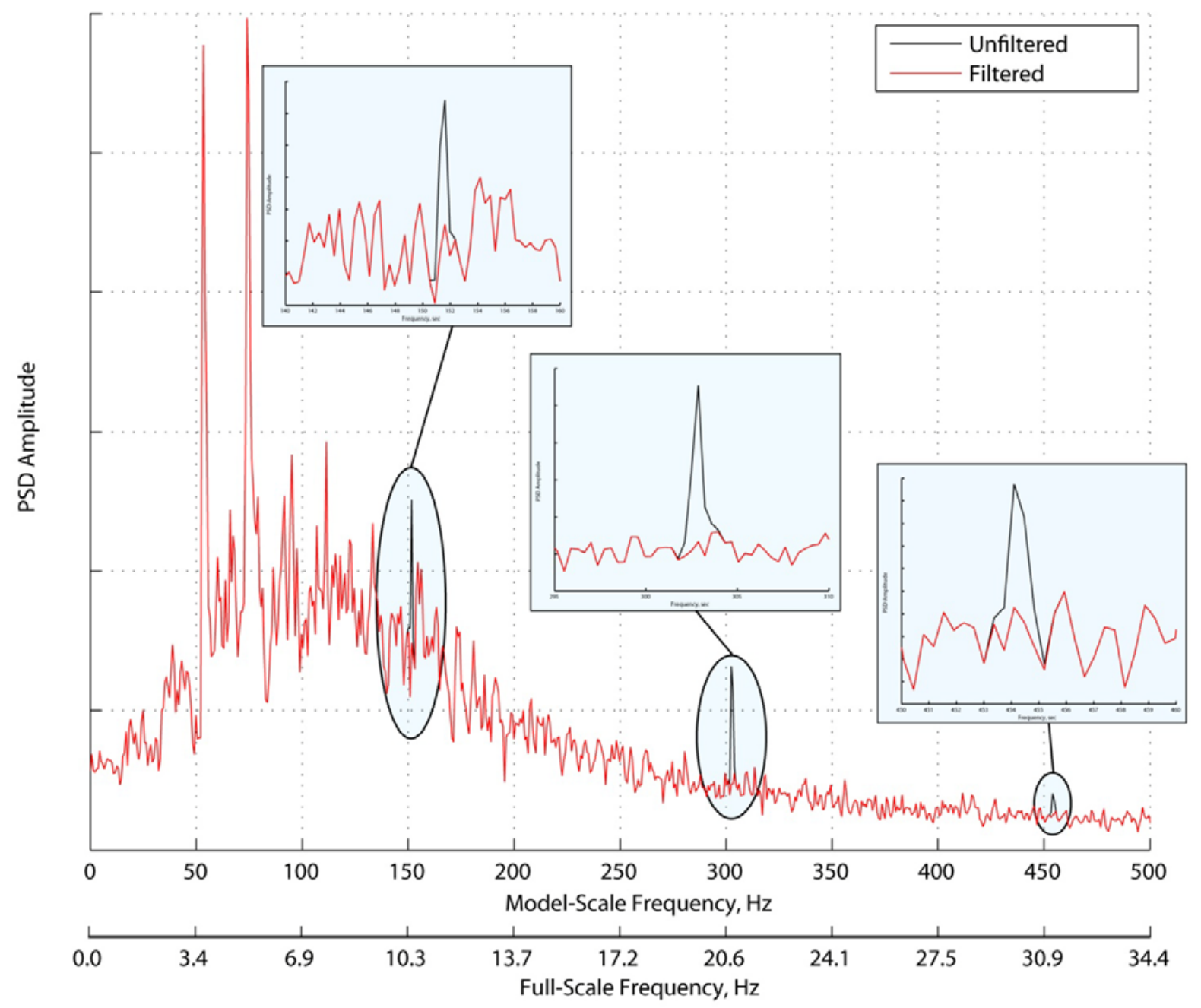

Figure 15. Effect of notch filtering technique on PSD of Ares I RBM buffet forcing function. 

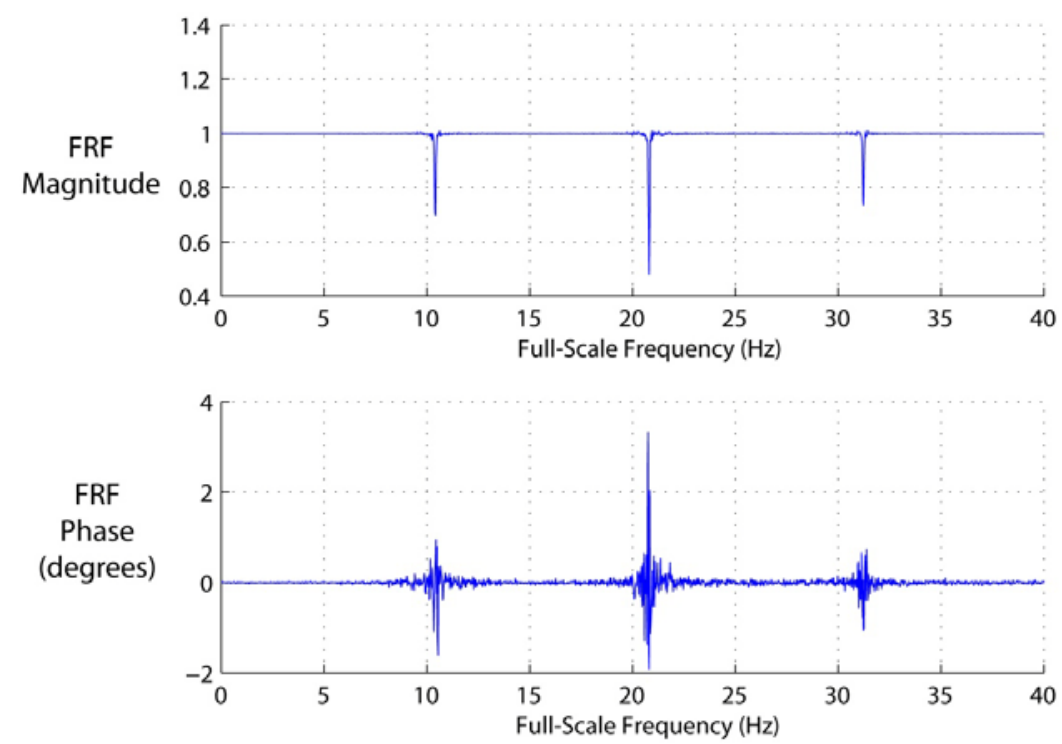

Figure 16. Frequency response function between filtered and unfiltered Ares I buffet forcing function.

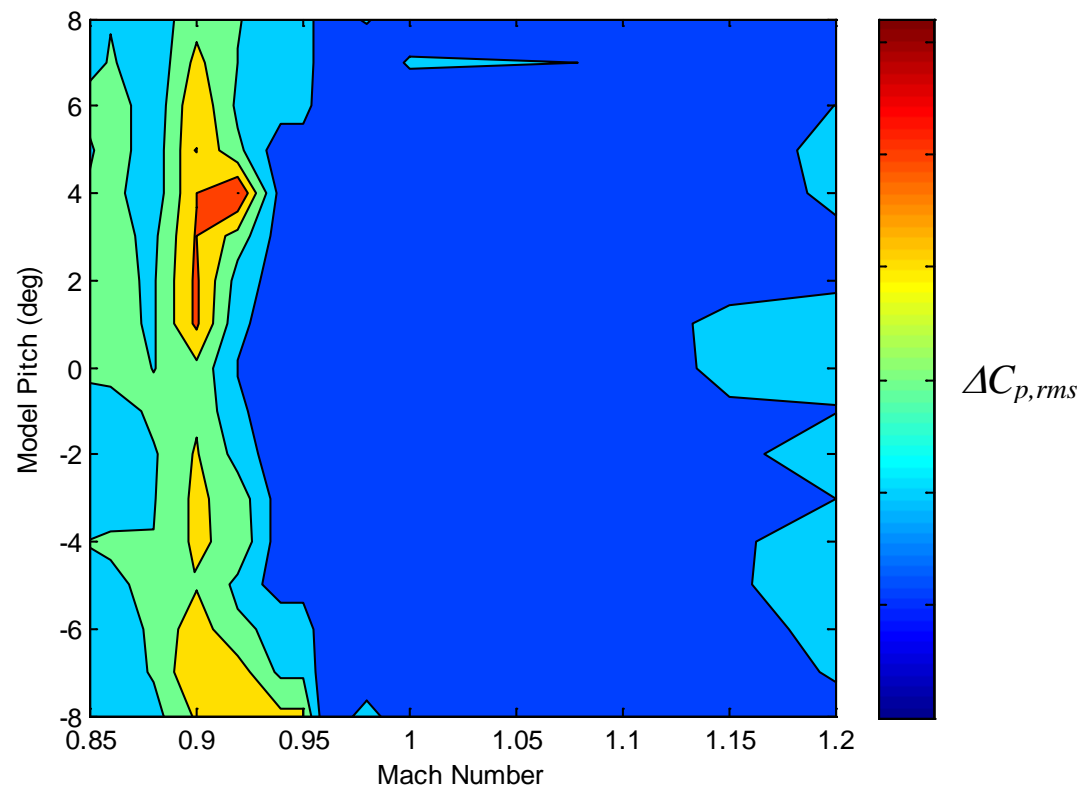

Figure 17. Ares I-X RBM contour plot of peak $\Delta C_{p, r m s}$ versus pitch and test section Mach number for the protuberance on the configuration in an R134a test medium. 


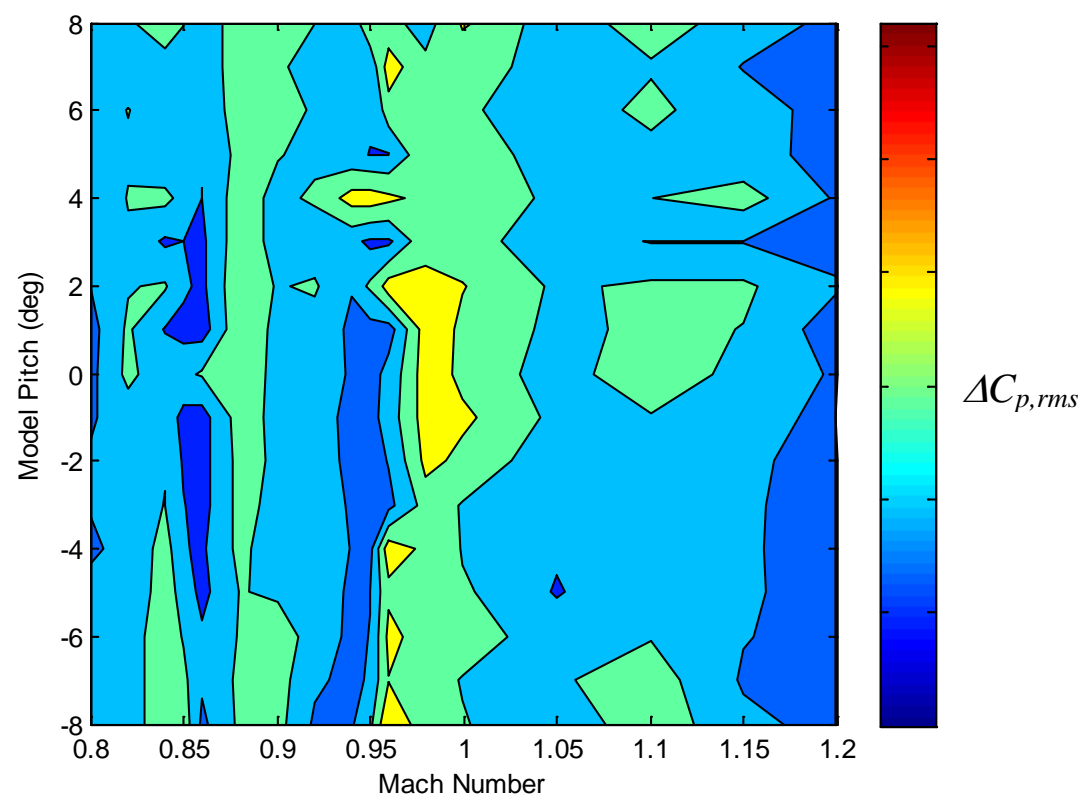

Figure 18. Ares I RBM contour plot of peak $\Delta C_{p, r m s}$ versus pitch and test section Mach number for the protuberance on the configuration in an R134a test medium.
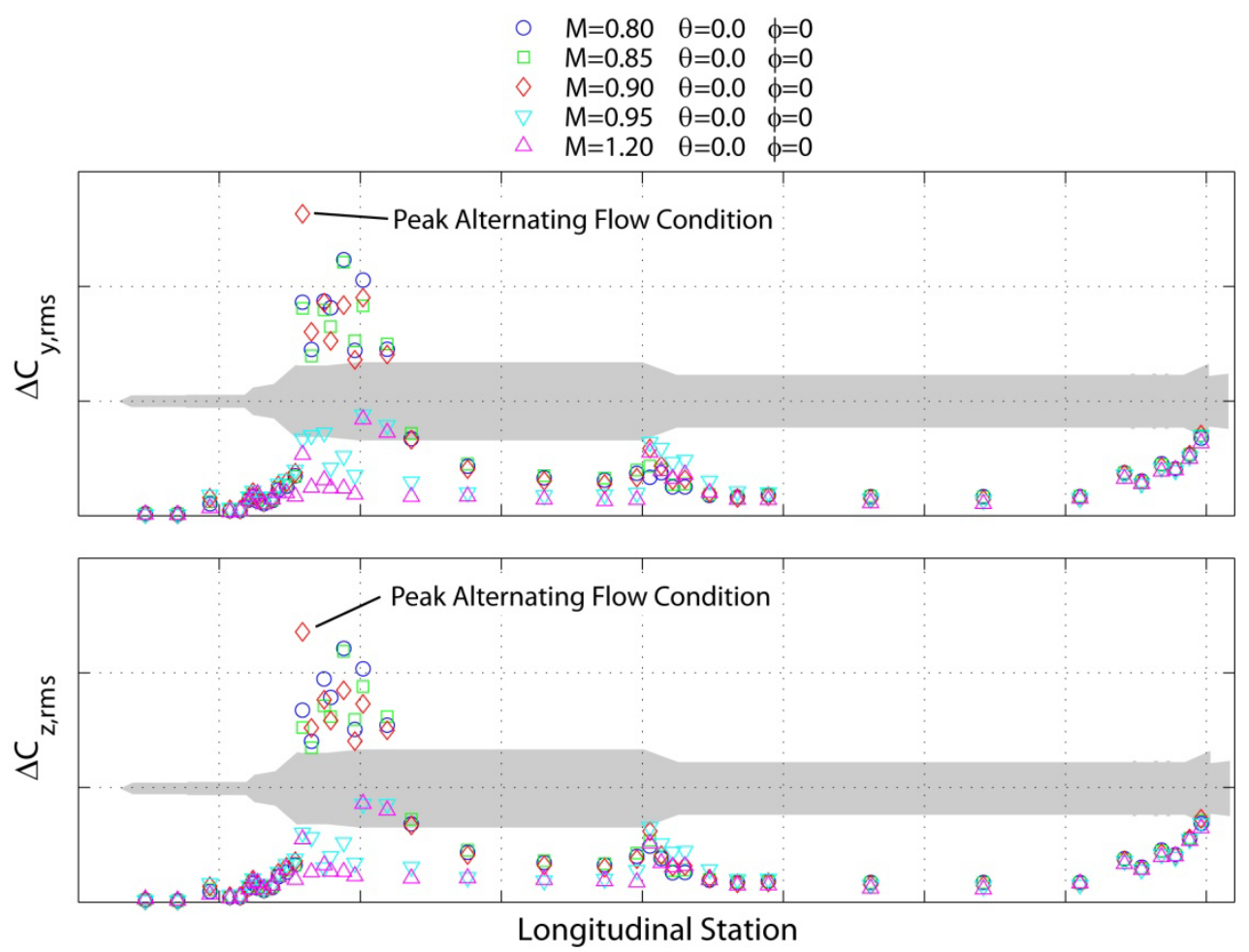

Figure 19. Ares I-X RBM $\Delta C_{y, r m s}$ and $\Delta C_{z, r m s}$ versus longitudinal vehicle station for Mach numbers between 0.80 and $1.20, \theta=0^{\circ}$, and $\phi=0^{\circ}$. 

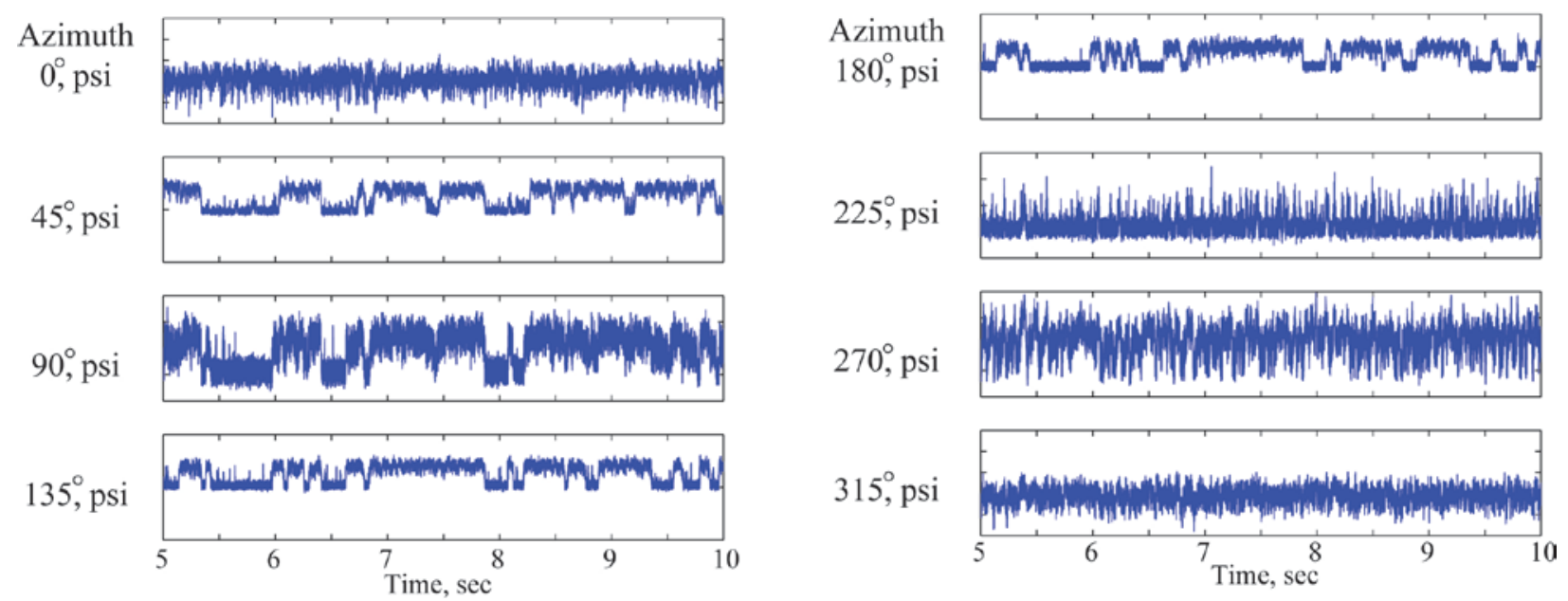

Figure 20. Ares I-X RBM pressure time histories illustrating alternating flow condition at Mach 0.90 at the $\mathrm{CM} / \mathrm{SM}$ cone-cylinder interface.

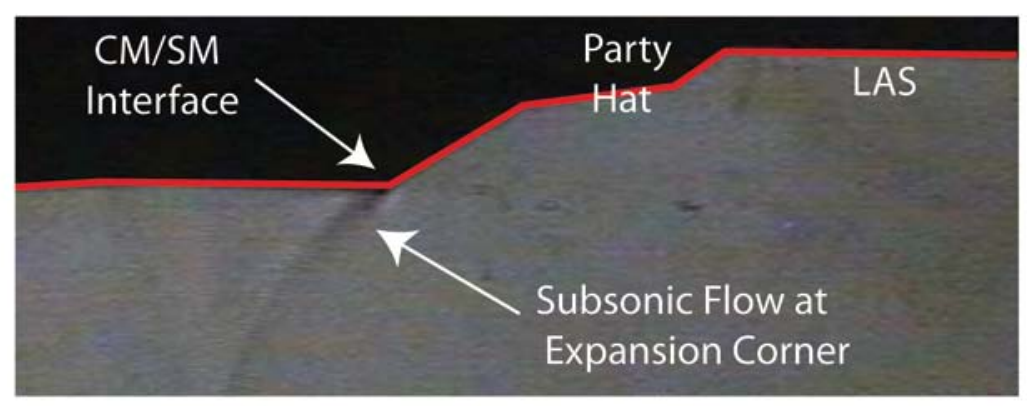

(a)

Expansion Corner

Alternating

Flow State \# 1

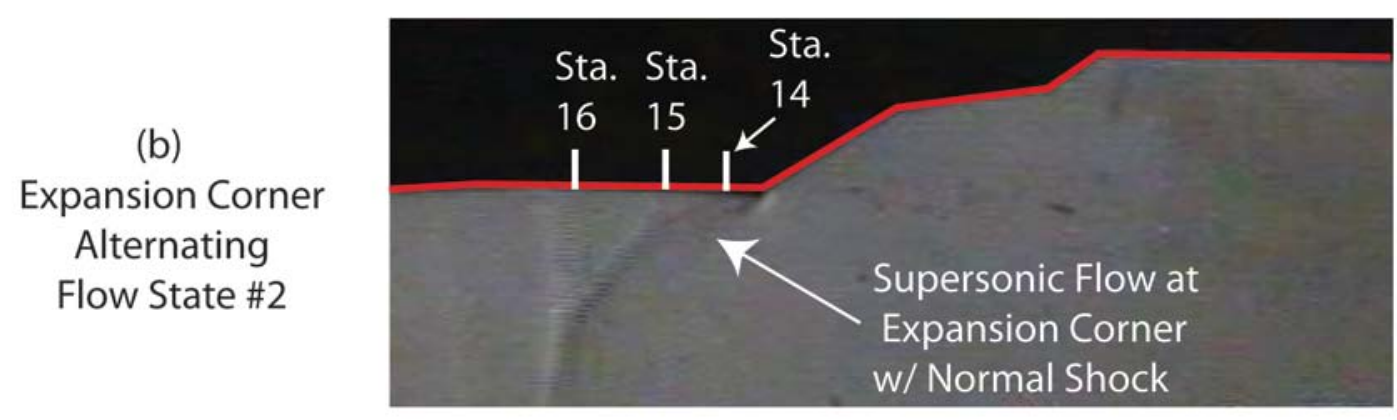

Figure 21. Shadowgraph images of Ares I-X alternating flow condition at Mach 0.9. 

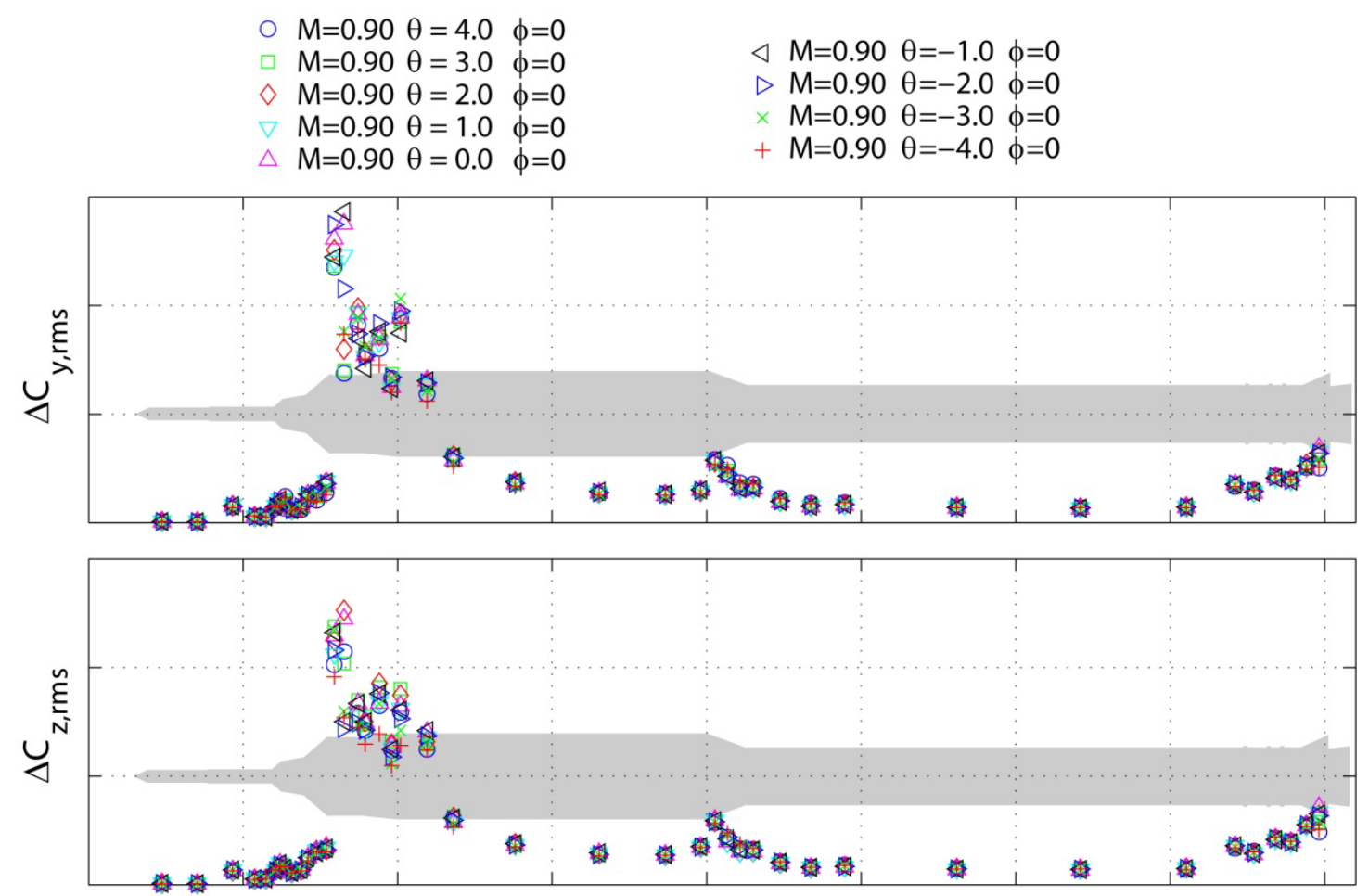

Longitudinal Station

Figure 22. Ares I-X RBM $\Delta C_{y, r m s}$ and $\Delta C_{z, r m s}$ versus longitudinal vehicle station for Mach number of $0.90, \theta=$ $-4^{\circ}$ to $+4^{\circ}$, and $\phi=0^{\circ}$.
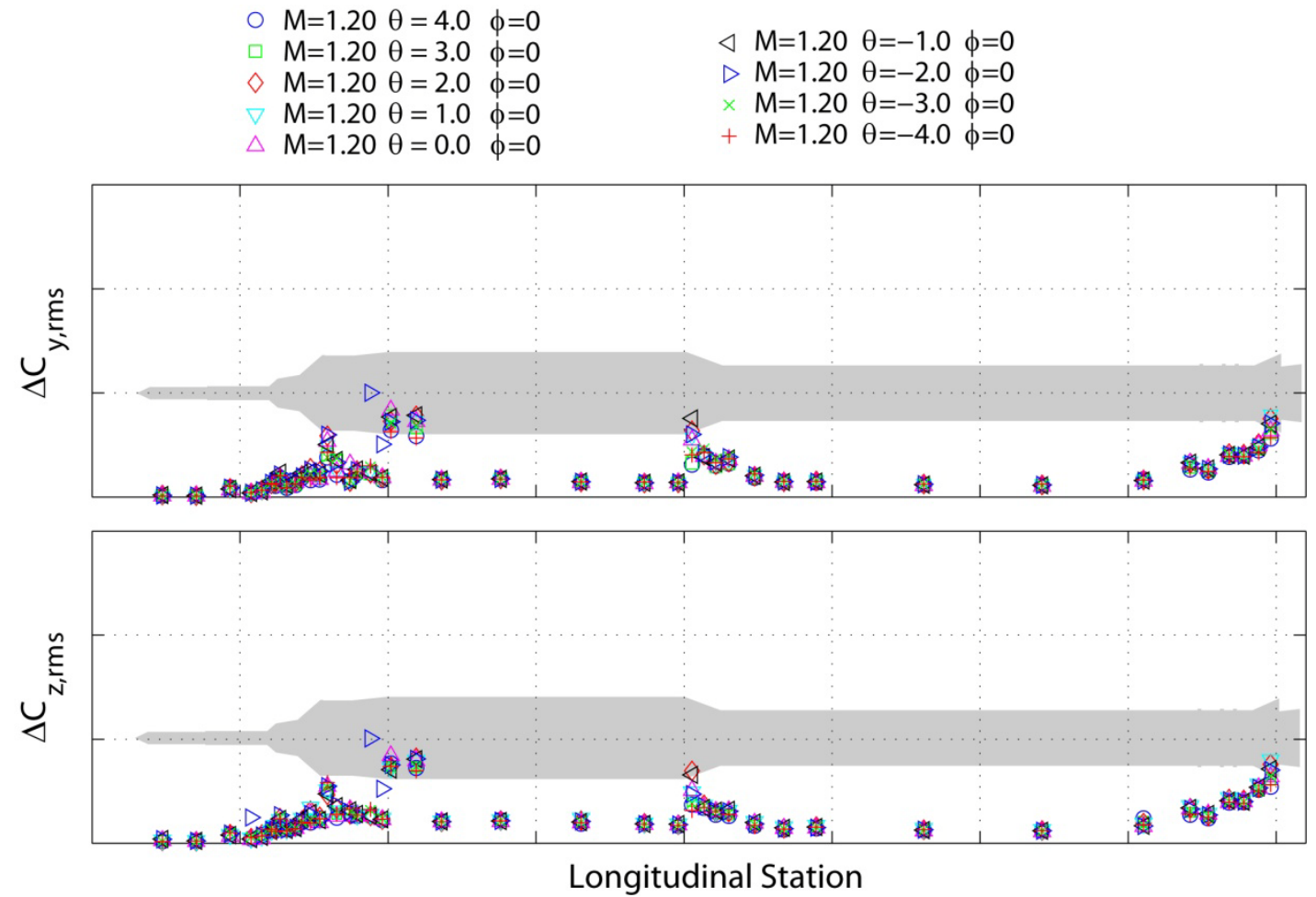

Figure 23. Ares I-X RBM $\Delta C_{y, r m s}$ and $\Delta C_{z, r m s}$ versus longitudinal vehicle station for Mach number of 1.2, $\theta=-$ $4^{\circ}$ to $+4^{\circ}$, and $\phi=0^{\circ}$. 

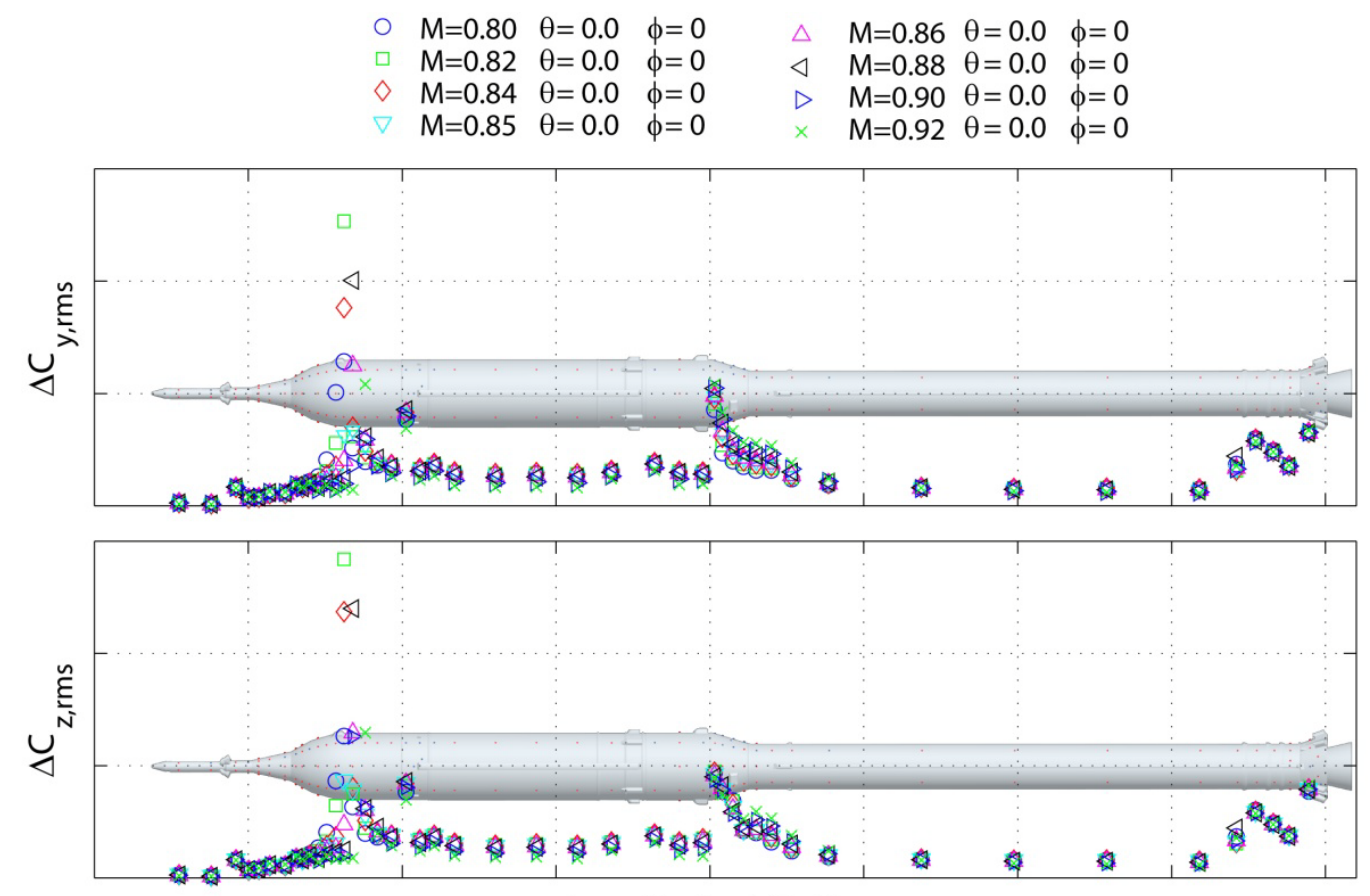

Longitudinal Station

Figure 24. Ares I RBM $\Delta C_{y, r m s}$ and $\Delta C_{z, r m s}$ versus longitudinal vehicle station for Mach numbers between 0.80 and 0.92, $\theta=0^{\circ}$, and $\phi=0^{\circ}$.
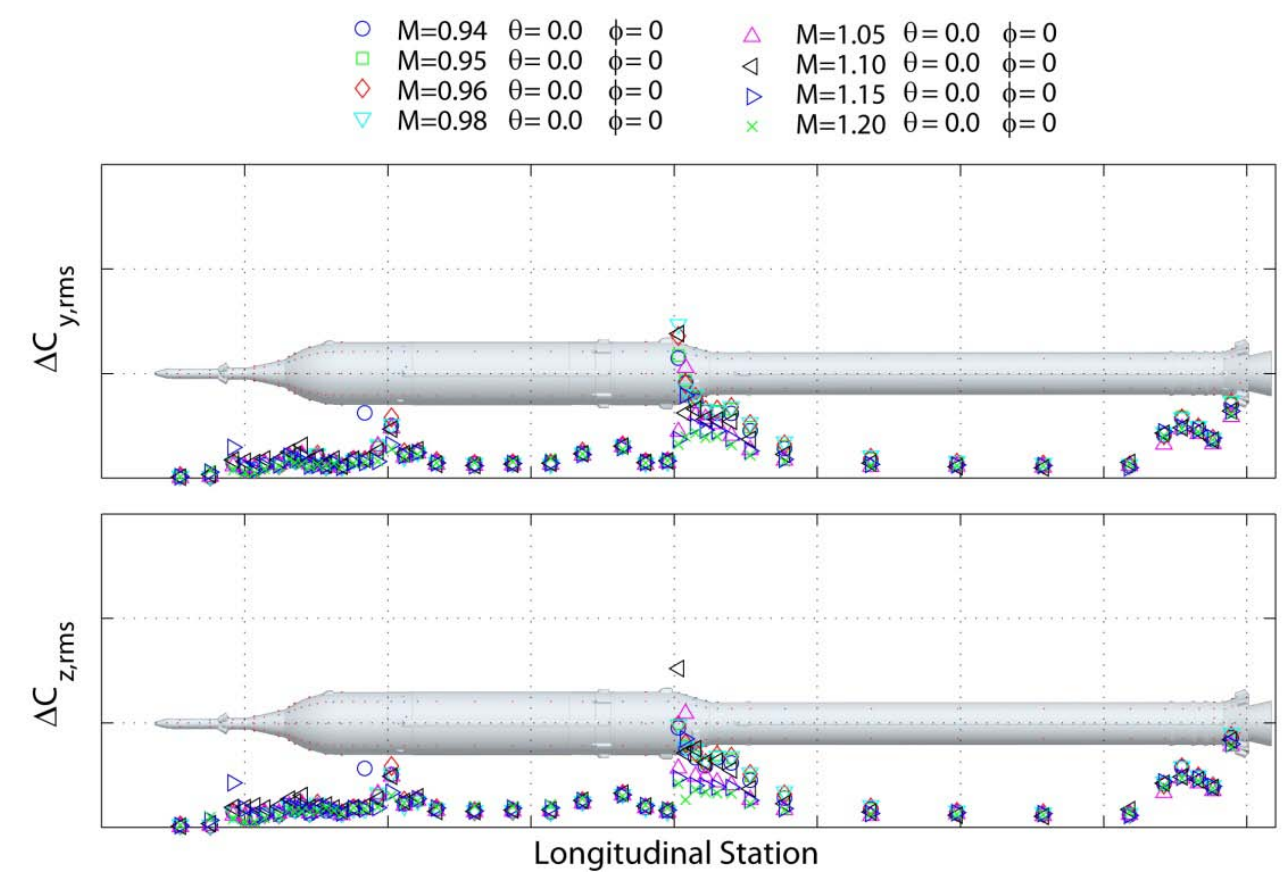

Figure 25. Ares I RBM $\Delta C_{y, \text { rms }}$ and $\Delta C_{z, \text { rms }}$ versus longitudinal vehicle station for Mach numbers between 0.94 and 1.20, $\theta=0^{\circ}$, and $\phi=0^{\circ}$. 

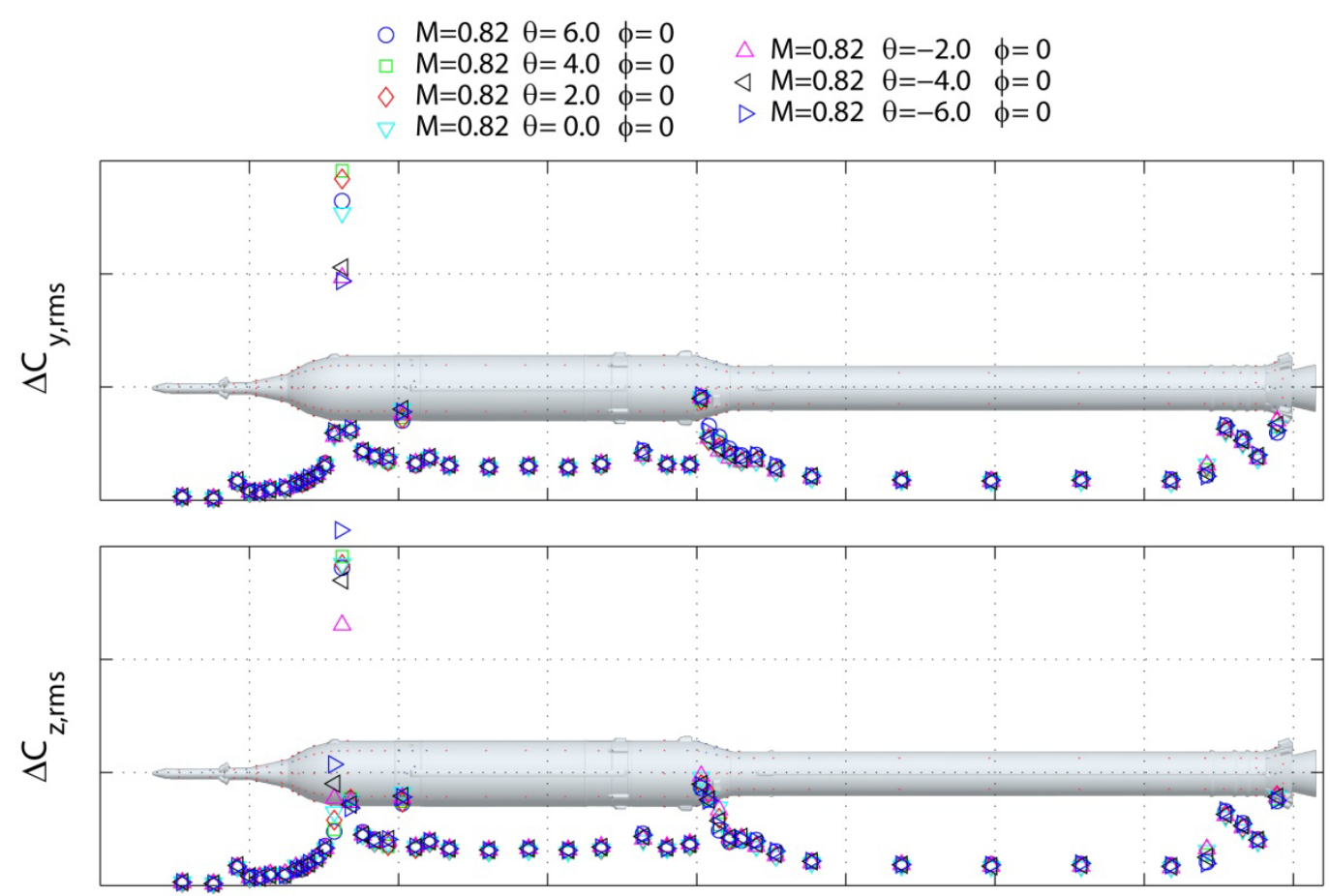

Longitudinal Station

Figure 26. Ares I RBM $\Delta C_{y, \text { rms }}$ and $\Delta C_{z, \text { rms }}$ versus longitudinal vehicle station for a Mach number of $0.82, \theta=$ $6^{\circ}$ to $+6^{\circ}$, and $\phi=0^{\circ}$.
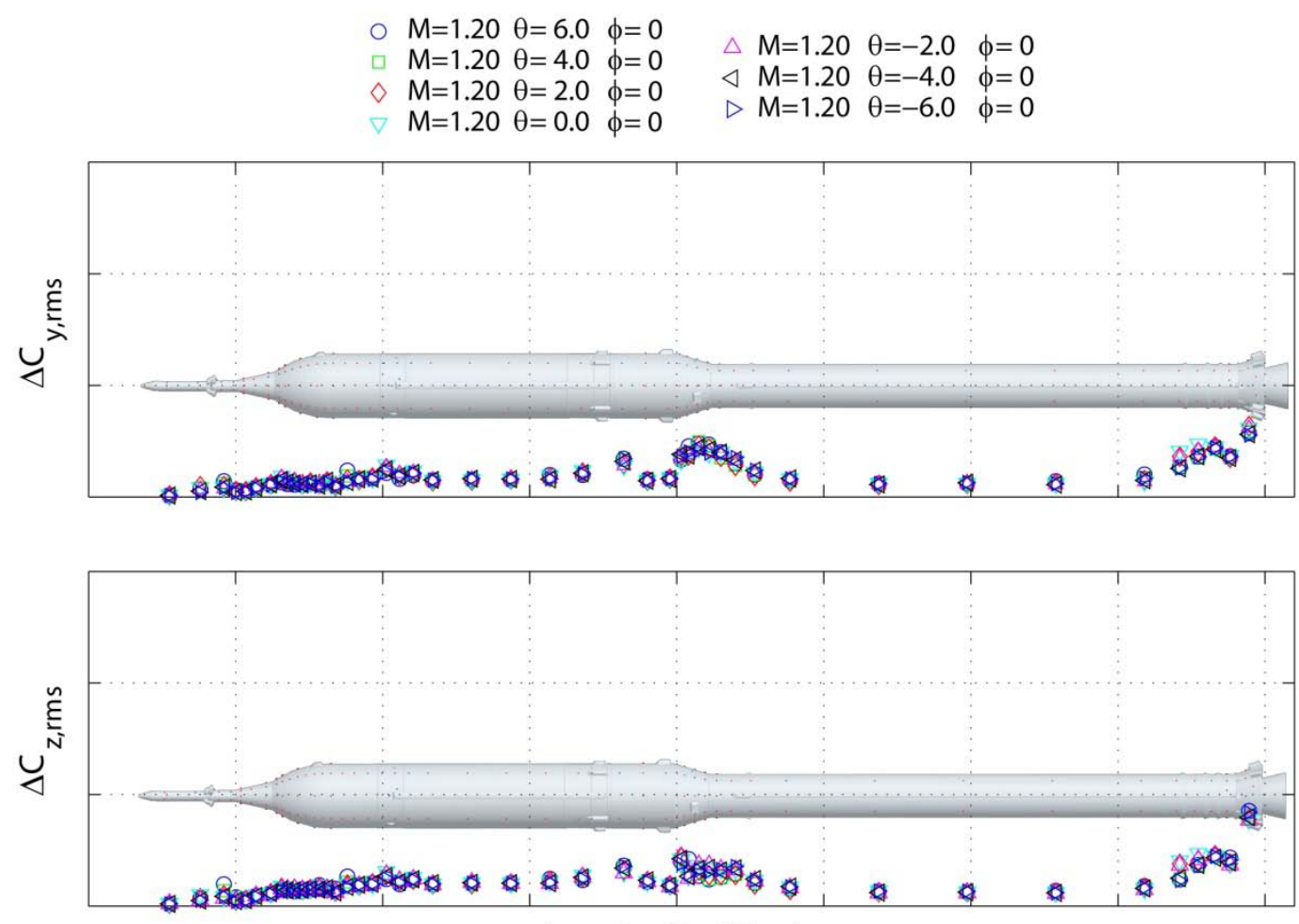

Longitudinal Station

Figure 27. Ares I RBM $\Delta C_{y, \text { rms }}$ and $\Delta C_{z, \text { rms }}$ versus longitudinal vehicle station for a Mach number of 1.20, $\theta=$ $6^{\circ}$ to $+6^{\circ}$, and $\phi=0^{\circ}$. 
American Institute of Aeronautics and Astronautics 